MR. ROMAIN FERON (Orcid ID : 0000-0001-5893-6184)

DR. CHRISTOPHE KLOPP (Orcid ID : 0000-0001-7126-5477)

DR. MATTHIAS STÖCK (Orcid ID : 0000-0003-4888-8371)

DR. ROBERT M. WATERHOUSE (Orcid ID : 0000-0003-4199-9052)

Article type : Resource Article

\title{
RADSex: a computational workflow to study sex determination using Restriction Site-Associated DNA Sequencing data
}

Romain Feron ${ }^{1,2,3^{*}}$, Qiaowei $\operatorname{Pan}^{1,2}$, Ming Wen ${ }^{1,4}$, Boudjema Imarazene ${ }^{1}$, Elodie Jouanno ${ }^{1}$, Jennifer Anderson ${ }^{1,5}$, Amaury Herpin ${ }^{1}$, Laurent Journot ${ }^{6}$, Hugues Parrinello ${ }^{6}$, Christophe Klopp ${ }^{7}$, Verena A. Kottler ${ }^{8}$, Alvaro S. Roco ${ }^{8}$, Kang Du ${ }^{9,10}$, Susanne Kneitz ${ }^{8}$, Mateus Adolfi10, Catherine A. Wilson ${ }^{11}$, Braedan McCluskey ${ }^{11}$, Angel Amores ${ }^{11}$, Thomas Desvignes ${ }^{11}$, Frederick W. Goetz ${ }^{12}$, Ato Takanashi ${ }^{13}$, Mari Kawaguchi ${ }^{13}, \mathrm{H}$. William Detrich $\mathrm{III}^{14}$, Marcos Oliveira ${ }^{15}$, Rafael Nobrega ${ }^{15}$, Takashi Sakamoto ${ }^{16}$, Masatoshi Nakamoto ${ }^{16}$, Anna Wargelius ${ }^{17}$, Ørjan Karlsen ${ }^{17}$, Zhongwei Wang ${ }^{8,18}$, Matthias Stöck ${ }^{19}$, Robert M. Waterhouse ${ }^{2,3}$, Ingo Braasch20, John $\mathrm{H}$. Postlethwait ${ }^{11}$, Manfred Schartl ${ }^{9,10}$ and Yann Guiguen ${ }^{1, *}$

\section{Affiliations}

1. INRAE, LPGP, 35000, Rennes, France

2. Department of Ecology and Evolution, University of Lausanne, 1015 Lausanne, Switzerland

3. Swiss Institute of Bioinformatics, 1015 Lausanne, Switzerland

This article has been accepted for publication and undergone full peer review but has not been through the copyediting, typesetting, pagination and proofreading process, which may lead to differences between this version and the Version of Record. Please cite this article as doi:

$\underline{10.1111 / 1755-0998.13360}$

This article is protected by copyright. All rights reserved 
4. State Key Laboratory of Developmental Biology of Freshwater Fish, College of Life Science, Hunan Normal University, Changsha, China

5. Department of Systematic Biology, EBC, Uppsala University, Norbyvägen 18D 75236 Uppsala, Sweden

6. Institut de Génomique Fonctionnelle, IGF, CNRS, INSERM, Univ. Montpellier, F-34094 Montpellier, France

7. SIGENAE, Mathématiques et Informatique Appliquées de Toulouse, INRAE, Castanet Tolosan, France

8. Physiological Chemistry, Biocenter, University of Wuerzburg, 97074 Wuerzburg, Germany

9. The Xiphophorus Genetic Stock Center, Department of Chemistry and Biochemistry, Texas State University, San Marcos, Texas, USA

10. Developmental Biochemistry, Biocenter, University of Wuerzburg, 97074 Wuerzburg, Germany

11. Institute of Neuroscience, University of Oregon, Eugene, OR 97403, USA

12. Environmental and Fisheries Sciences Division, Northwest Fisheries Science Center, National Marine Fisheries Service, National Oceanic and Atmospheric Administration, 2725 Montlake Blvd East, Seattle, WA 98112, USA

13. Department of Materials and Life Sciences, Faculty of Science and Technology, Sophia University, 7-1 Kioi-cho, Chiyoda-ku, Tokyo, 102-8554 Japan

14. Department of Marine and Environmental Sciences, Marine Science Center, Northeastern University, Nahant, Massachusetts 01908

15. Reproductive and Molecular Biology Group, Department of Structural and Functional Biology, Institute of Biosciences, São Paulo State University, Botucatu, São Paulo, Brazil

16. Department of Aquatic Marine Biosciences, Tokyo University of Marine Science and Technology, Tokyo 108-8477, Japan

17. Institute of Marine Research, P.O. Box 1870, Nordnes, NO-5817, Bergen, Norway

18. Institute of Hydrobiology, Chinese Academy of Sciences, Beijing, China.

19. Leibniz-Institute of Freshwater Ecology and Inland Fisheries, IGB, Berlin, Germany

20. Department of Integrative Biology, Michigan State University, East Lansing, MI, USA

*Corresponding authors: yann.guiguen@inrae.fr; + 3324850 09; romainferon91@gmail.com;

This article is protected by copyright. All rights reserved 


\section{ABSTRACT}

The study of sex determination and sex chromosome organisation in non-model species has long been technically challenging, but new sequencing methodologies now enable precise and highthroughput identification of sex-specific genomic sequences. In particular, Restriction Site-Associated DNA Sequencing (RAD-Seq) is being extensively applied to explore sex determination systems in many plant and animal species. However, software specifically designed to search for and visualize sex-biased markers using RAD-Seq data is lacking. Here, we present RADSex, a computational analysis workflow designed to study the genetic basis of sex determination using RAD-Seq data. RADSex is simple to use, requires few computational resources, makes no prior assumptions about the type of sex-determination system or structure of the sex locus, and offers convenient visualization through a dedicated $\mathrm{R}$ package. To demonstrate the functionality of RADSex, we reanalyzed a published dataset of Japanese medaka, Oryzias latipes, where we uncovered a previously unknown $\mathrm{Y}$ chromosome polymorphism. We then used RADSex to analyze new RAD-Seq datasets from 15 fish species spanning multiple taxonomic orders. We identified the sex determination system and sex-specific markers in six of these species, five of which had no known sex-markers prior to this study. We show that RADSex greatly facilitates the study of sex determination systems in non-model species thanks to its speed of analyses, low resource usage, ease of application, and visualization options. Furthermore, our analysis of new datasets from 15 species provides new insights on sex determination in fish.

Keywords: sex determination, RAD-Sequencing, fish, computational workflow, visualization

\section{INTRODUCTION}

Sexual reproduction is widespread in animals (Goodenough \& Heitman, 2014) and, in gonochoristic species, involves segregation of male and female gonadal functions into separate individuals for their entire lives (Bachtrog et al., 2014). Males produce small, mobile gametes while females produce large, immobile gametes, and this difference has fueled divergent evolution of morphological, physiological, and behavioral traits between the two sexes. Individuals acquire sexspecific traits during sexual development, which starts with sex determination, the process that controls whether an individual develops male or female reproductive organs. Sex determination can 
be triggered by genetic factors (Genetic Sex Determination, GSD), like in virtually all mammals and birds (Bachtrog et al., 2014); by environmental factors (Environmental Sex Determination, ESD), like temperature in many non-avian reptiles (Pezaro, Doody, \& Thompson, 2017); or a combination of the both, as in several fish species (Piferrer, Blázquez, Navarro, \& González, 2005). When genetic factors are involved, sex determination is entirely or partially controlled by one or multiple master sex determining (MSD) genes located on sex chromosomes. Genetic sex determination systems can involve male heterogamy $(X X / X Y$ and $X X / X 0)$, female heterogamy ( $Z Z / Z W$ and $Z Z / Z 0)$, or multiple loci on different chromosomes (polygenic systems) (Bachtrog et al., 2014). In male- and femaleheterogametic systems, sex chromosomes can be morphologically different, i.e. heteromorphic, or similar, i.e. homomorphic (Bachtrog et al., 2014). Initially, the overwhelming majority of knowledge on the structure and evolution of sex chromosomes came from studies in mammals (Wallis, Waters, \& Graves, 2008) and in Drosophila (Salz \& Erickson, 2010), where it has been observed that suppression of recombination between the $X$ and $Y$ chromosomes led to degeneration of the $Y$. These findings have spawned both theoretical and empirical interest in sex chromosomes as models to study the consequences of recombination suppression and associated processes (Bachtrog, 2008; Charlesworth \& Charlesworth, 2000; Corcoran et al., 2016; Doorn \& Kirkpatrick, 2007; L. Gu, Walters, \& Knipple, 2017; Huylmans, Macon, \& Vicoso, 2017; Muyle et al., 2012; Peichel et al., 2004). More recent studies investigating sex chromosomes in insects (Blackmon, Ross, \& Bachtrog, 2017), non-avian reptiles (Modi \& Crews, 2005), amphibians (Miura, 2017), and fishes (Kikuchi \& Hamaguchi, 2013) have found many homomorphic sex chromosomes displaying varying levels of differentiation, with the extreme case of a sex locus restricted to allelic variation of a single nucleotide as reported in the Japanese pufferfish (Kamiya et al., 2012). These results called into question the single unified concept of sex chromosome evolution and highlighted the importance of obtaining a broader understanding of sex determination and sex chromosomes in many species across the tree of life. The first step in this process is identifying sex-specific genomic sequences, i.e., sequences from the sex locus that are found in only one of the two sexes because of allelic divergence between the sex chromosomes or because of large insertions in the hemizygous chromosome. These sexspecific sequences can then be aligned to a reference genome to locate the sex locus, identify candidate MSD genes and other genes involved in sex determination, and characterize patterns of differentiation between the sex chromosomes. In addition, facilitating the identification of such sequences has practical applications: sex is an important factor in ecological (Benestan et al., 2017) and conservation studies (Ancona, Dénes, Krüger, Székely, \& Beissinger, 2017), as well as 
agriculture (Al-Ameri, Al-Qurainy, Gaafar, Khan, \& Nadeem, 2016; Liao, Yu, \& Ming, 2017; Spigler, Lewers, Main, \& Ashman, 2008) and animal production (Dan, Mei, Wang, \& Gui, 2013; Yano et al., 2013), yet the sex of an individual cannot always be easily determined by non-invasive methods.

Until recently, discovery of sex loci was not readily feasible mainly due to technical barriers preventing the precise and high-throughput identification of sex-specific genomic sequences, especially in non-model species. Genetic mapping has for a long time been the traditional approach for studying genetic sex determination (Palmer, Rogers, Dean, \& Wright, 2019), but recent advances in sequencing technologies enable the exploration of a much broader spectrum of taxa by directly comparing genomes from phenotypically distinct males and females. In essence, this process is akin to comparing genomic differences between two populations or between a mutant and a wild type genotype, and therefore methods from molecular genetics, population genetics, and ecology can be applied. A popular representational approach to comparing the genetics of populations is Restriction Site-Associated DNA Sequencing, or RAD-Seq (Andrews, Good, Miller, Luikart, \& Hohenlohe, 2016). RAD-Seq generates short sequences for a small but consistent fraction of the genome and thus allows the sequencing and comparison of multiple individuals from several populations at relatively low cost (Davey et al., 2011) and without requiring additional genomic resources. RAD-Seq has been successfully used to identify sex-specific sequences in non-model species from diverse taxa, including fish (Drinan, Loher, \& Hauser, 2018), amphibians (Bewick et al., 2013), non-avian reptiles (Gamble, 2016; Gamble et al., 2017, 2015, 2018; Gamble \& Zarkower, 2014; S. V. Nielsen, Banks, Diaz, Trainor, \& Gamble, 2018; Nielsen, Daza, Pinto, \& Gamble, 2019), invertebrates (Carmichael et al., 2013; Mathers et al., 2015; Pratlong et al., 2017), and plants (Kafkas, Khodaeiaminjan, Güney, \& Kafkas, 2015), and to identify the sex chromosomes and the sex locus in some of these species (Wilson et al., 2014). Although dedicated pipelines have been developed to analyze RAD-Seq data specifically for sex determination (Gamble \& Zarkower, 2014), most of the above mentioned studies have used Stacks (Catchen, Hohenlohe, Bassham, Amores, \& Cresko, 2013; Catchen, Amores, Hohenlohe, Cresko, \& Postlethwait, 2011; Hohenlohe, Catchen, \& Cresko, 2012; Rochette \& Catchen, 2017) to cluster RAD-Seq reads into polymorphic markers subsequently filtered with custom in-house scripts. Stacks, however, requires substantial computational resources to run on large datasets and depends on multiple parameters that can greatly influence the outcome of the analysis (Paris, Stevens, \& Catchen, 2017; Rodríguez-Ezpeleta et al., 2016; Shafer et al., 2018), for instance, in the context of sex determination, the classification of a marker as a sex-specific sequence (e.g., from a large sex-specific insertion) or as a sex-specific allele (e.g., an allele specific 
to one sex in a polymorphic locus) (Utsunomia et al., 2017). In addition, the current visualization tools for RAD-Seq data make the interpretation of results from some datasets difficult, for instance, datasets containing strong population structure, individuals with mis-assigned phenotypic sex, or sex-bias in sequencing depth.

To overcome these limitations, we developed RADSex, a RAD-Seq data analysis computational workflow specifically designed to study sex determination. RADSex is simple to use, makes no assumptions about whether sex-biased markers are sex-biased sequences or sex-biased alleles in a polymorphic locus, requires few resources, and offers helpful visualization tools in the form of an $\mathrm{R}$ package. To demonstrate the relevance of RADSex, we analyzed a previously published dataset from the Japanese medaka, Oryzias latipes, for which we replicated previous findings, including the identification of two sex-reversed individuals, and we uncovered a previously overlooked Y-specific polymorphism in the sequenced population. We then used RADSex to analyze new datasets that we generated from 15 fish species spanning multiple systematic orders. We identified the sex-determination system in six of these species as well as multiple sex markers, and we identified the sex chromosome in one species for which a reference genome was available. Our results show that RADSex is well-suited to study genetic sex-determination in non-model species thanks to its speed, resource usage, and ease of use. Furthermore, our multi-species analysis provides insights into the genetic mechanisms of sex-determination in fishes and highlights some of the limitations of RAD-Seq in detecting small sex loci.

\section{MATERIAL AND METHODS}

\section{Overview of the RADSex analysis workflow}

The underlying principle of RADSex is to group identical RAD-Seq reads from all individuals in a dataset into non-polymorphic markers, and then consider the presence or absence of each marker in each individual. Markers created by RADSex thus differ from that of other software such as Stacks (Catchen et al., 2011) which typically attempt to reconstruct genotypes from polymorphic markers. The RADSex workflow (Fig. 1) includes the command-line software radsex implemented in C++ (https://github.com/SexGenomicsToolkit/radsex) and the $\mathrm{R}$ package sgtr (https://github.com/SexGenomicsToolkit/sgtr) to visualize results from radsex. The radsex software 
includes several commands, starting with process, a data processing command that takes as input a set of demultiplexed reads, i.e., one fasta or fastq file containing short reads of consistent size from either single or double digest RAD-seq protocols for each individual. The other commands perform analyses using the output of process: distrib computes the distribution of marker presence between males and females; signif extracts all markers significantly associated with sex; map aligns markers to a reference genome sequence; depth computes the distribution of marker depths in each individual; freq computes the distribution of marker presence in all individuals; and subset filters markers based on presence in males and females.

The first step of the workflow is to compute a table of marker depths. The process command creates a table with identifier, sequence, and depth in each individual for all unique sequences in the entire dataset. This table summarizes all the sequence information present in the dataset, and each row constitutes a marker for radsex (Supp. Fig. 1). Sequences are read from each individual reads file and are stored in a hash table with the sequence as key and an array of individual sequencing depths as values. Each time a sequence is read from an individual read file, its depth is incremented in the corresponding value column of the hash table. By design, and in contrast to markers typically generated by other tools, markers from radsex are not polymorphic. Instead, each allele at a polymorphic locus is treated as a separate marker. The table created by process is stored in a plain text tabulated file (Supp. Table 1) and is the main input data for all subsequent analyses performed by radsex. The process command can be parallelized, each thread processing one individual input file at a time.

The next step is to identify markers significantly associated with sex. The distrib command uses the table of marker depths generated with process to compute the distribution of markers between males and females. Phenotypic sex for each individual is given by a user-supplied tabulated file with the identifier of each individual in the first column and its phenotypic sex in the second column. In all radsex commands, a marker is defined as present in a given individual if the depth of this marker in this individual is higher than a user-specified threshold given by the parameter --mindepth (or -d) (Supp. Fig. 1). Following this definition, the distrib command enumerates marker presence in every possible combination of number of males and number of females (e.g., for a

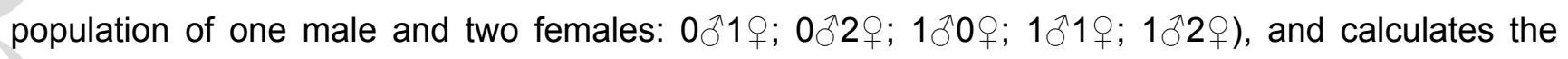
probability of association with sex for each combination using Pearson's chi-squared test of independence. Yates' correction for continuity is applied to account for low individual numbers in some of the tests. The associated p-value is obtained from the Cumulative Density Function of the 
chi-squared distribution with one degree of freedom, as implemented in Samtools (Li et al., 2009). Bonferroni correction is applied by multiplying the p-value by the total number of markers present in at least one individual. Markers for which association with sex is significant, i.e., $p<0.05$ (or a userspecified threshold) after Bonferroni correction, can be obtained with the signif command and exported either in the same format as the marker depths table or in fasta format. The results of distrib (Supp. Table 2) can be visualized using the radsex_distrib() function from sgtr, which generates a tile plot with the number of males on the horizontal axis, the number of females on the vertical axis, and the number of markers represented by a tile's color for each number of males and females. Tiles for which association with sex is significant are highlighted with a red border. Simplified examples of tile plots for several sex-determining systems are presented in Fig. 2.

Next, RADSex can filter and cluster markers based on depth across individuals to identify consistent absence or presence of markers in some individuals. Markers can be filtered based on presence in males and females using the radsex subset command and exported in the same format as the table of marker depths (Supp. Fig. 1). After extracting a subset of markers, a heatmap showing the depth of each marker in each individual can be generated using the radsex_markers_depth() function from sgtr. This function optionally allows the user to cluster both individuals and markers based on depth values and display the resulting cladograms along the heatmap. Distances are computed using the base $\mathrm{R}$ dist function and clustering is performed with the base $\mathrm{R}$ hclust function; both distance calculation (e.g., euclidean, maximum, binary ...) and clustering methods (e.g., complete, average, centroid ...) can be specified by the user. An application of the subset command is the detection of outliers in the sequenced population, e.g., sex-reversed individuals or individuals with misassigned sex. In such cases, some markers may have biased presence in individuals from one sex without being statistically associated with phenotypic sex. When clustering individuals based on depth for these sex-biased markers, real outliers will be grouped with individuals from the other sex (see Fig. 4.B for an example from a real dataset). Markers obtained with the signif command can also be used as input for the radsex_markers_depth() function from sgtr if they were exported as a table of marker depths.

When a genome assembly is available, markers can be aligned to it using the radsex map command to locate genomic regions differentiated between sexes. Markers obtained from either the process, signif, or subset commands are aligned to the genome using the BWA mem library (Li, 2013), and only markers uniquely aligned with a mapping quality higher than a user-specified threshold are retained. Two metrics are computed for each retained marker: 1) the probability of 


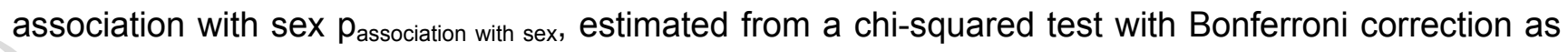
described for the distrib command, and 2) the sex-bias $(S)$, defined as $S=\hat{\sigma} / \hat{o}$ total $-q / q$ total, where $\hat{\delta}$ and $q$ are the number of males and females in which the marker is present, and $\hat{\sigma}_{\text {total }}$ and $\Phi_{\text {total }}$ are the total number of males and females in the population, inferred from the user-supplied sex information file. Sex-bias thus ranges from -1 for a marker present in all females and absent from all males to +1 for a marker present in all males and absent from all females, and is zero for a marker present in the same proportion of males and females. Using the output of radsex map (Supp. Table 3), the radsex_map_circos() function of sgtr generates a circular plot in which sex-bias, on the top track, and $-\log _{10}\left(p_{\text {association with sex }}\right)$, on the bottom track, are plotted against genomic position using R's circlize package (Gu, Gu, Eils, Schlesner, \& Brors, 2014). Each sector of the plot represents a linkage group, with the last sector containing unplaced scaffolds, which are important to include due to frequent assembly problems with sex chromosomes. Each metric can also be displayed in a Manhattan plot using the radsex_map_manhattan() function; in addition, a linear plot showing both metrics for a given genomic region can be generated using the radsex_map_region() function from sgtr.

Finally, radsex provides two commands to assess the distribution of marker depths in the entire dataset. First, the freq command computes the number of individuals in which each marker is present for a given minimum depth value and generates a count table of markers present in each number of individuals. Second, the depth command computes the minimum, maximum, median, and average marker depth for each individual using markers present in more than $75 \%$ of individuals (or a user-specified threshold). Results from radsex freq and radsex depth can be visualized with the radsex_freq() and radsex_depth() functions from sgtr.

\section{Sample collection}

General information on the different species, species collectors, and samples is given in Supp. Table 5. Detailed information on sample collection for each dataset is provided in the supplementary material (Supplementary Methods 1). 


\section{Genomic DNA (gDNA) extraction}

Genomic DNA (gDNA) of Lepisosteus oculatus, Plecoglossus altivelis, Gadus morhua, Notothenia rossii, Sander vitreus, Tinca tinca, Carassius auratus, and all Danios were extracted from fin clips stored in ethanol using NucleoSpin Kits for Tissue (Macherey-Nagel, Duren, Germany) following the producer's protocol. Genomic DNA of Gymnotus carapo, Gymnocorymbus ternetzi, Hippocampus abdominalis, and Poecilia sphenops were extracted from individual fin clips stored in ethanol or from pooled organs of individual fish for $P$. sphenops with a phenol/chloroform protocol. Fin clips or tissues were lysed in $1 \mathrm{ml}$ extraction buffer $(0.1 \mathrm{M}$ EDTA pH 8, 0.2\% SDS, $0.2 \mathrm{M} \mathrm{NaCl})$ containing $200 \mu \mathrm{g} / \mathrm{ml}$ proteinase $\mathrm{K}$ for three hours at $80^{\circ} \mathrm{C}$, and $500 \mu \mathrm{L}$ phenol was added to each sample. After mixing and incubating at room temperature for $10 \mathrm{~min}, 500 \mu \mathrm{L}$ chloroform/isoamyl alcohol (24:1) was added. Samples were incubated with occasional inversion at room temperature for 10 min and then centrifuged for $10 \mathrm{~min}$ at $5000 \mathrm{~g}\left(5^{\circ} \mathrm{C}\right)$. The upper layer was transferred to a new tube and one volume of chloroform/isoamyl alcohol (24:1) was added. The samples were kept at room temperature for $10 \mathrm{~min}$ with occasional inverting, and subsequently centrifuged for $10 \mathrm{~min}$ at $5000 \mathrm{~g}\left(5^{\circ} \mathrm{C}\right)$. The supernatant was transferred to a glass vial on ice and 2.5 times the sample volume of cold $100 \%$ ethanol was carefully added. The DNA was spooled with a glass rod and dissolved in TE buffer $\mathrm{pH} 8$. All gDNA concentrations were quantified with a NanoDrop ND2000 spectrophotometer (Thermo scientific, Wilmington, Delaware) and/or Qubit (ThermoFisher, France) before processing gDNA for RAD libraries construction.

\section{RAD-Sequencing and demultiplexing of RAD-Seq reads}

RAD libraries were constructed for each species from individual fish gDNA using a single restriction enzyme, Sbfl, following the standard protocol as previously described (Amores, Catchen, Ferrara, Fontenot, \& Postlethwait, 2011). Libraries were sequenced as single end 100 bp reads on one lane of Illumina HiSeq 2500. Quality control and demultiplexing of the reads was performed with the process_radtags.pl wrapper script from Stacks version 1.44 (Catchen et al., 2011) using the following options: discard reads with low quality scores $(-q)$, remove any reads with an uncalled base $(-c)$, and rescue barcodes and RAD-seq reads (-r). Demultiplexing results for each species are summarized in Supp. Table 7. 


\section{Performance measurements}

Runtime and peak memory usage of radsex (version 1.0.0) was measured with the "benchmark" directive of Snakemake (Mölder et al., 2021) on the Genotoul computational platform (Toulouse, France) using 4 threads. Performance was measured for the process, distrib, and signif commands run sequentially with a minimum depth of 1 , because this value would require the longest runtime and memory.

\section{Software used in the analyses}

All analyses were performed using version 1.1.2 of radsex and figures were generated using version 1.1.2 of the sgtr $\mathrm{R}$ package in $\mathrm{R}$ version 3.5.2. Both radsex and sgtr are released under a GPLv3 license; releases and source code are available at https://github.com/SexGenomicsToolkit/radsex for radsex and https://github.com/SexGenomicsToolkit/sgtr for sgtr. Both radsex and sgtr are also available on the conda channel Bioconda. A complete documentation for RADSex is available at https://sexgenomicstoolkit.github.io/html/radsex/introduction.html.

\section{RESULTS}

\section{Performance of RADSex}

The runtime and peak memory usage of radsex process, distrib, and signif were measured on datasets from 15 fish species that we generated for this study. Apart from one outlier dataset, Danio albolineatus, RADSex's runtime was linearly and positively correlated to the number of RADseq reads, ranging from 276 seconds for Tinca tinca, whose dataset contained 120 M. RAD-seq reads, to 1240 seconds (20 min and $40 \mathrm{~s}$ ) for Sander vitreus, whose dataset contained $\sim 232 \mathrm{M}$. RAD-seq reads (Fig. 3.A). Runtime and peak memory usage for all datasets are provided in Supp. Table 4. RADSex's peak memory usage was also linearly and positively correlated to the number of RAD-seq reads, ranging from $2.2 \mathrm{~Gb}$ for Poecilia sphenops to $12.24 \mathrm{~Gb}$ for G. ternetzi (Fig. 3.B). The high runtime and memory usage of radsex for the Danio albolineatus dataset is related to an extremely high number of markers found in only one individual, which could indicate low sequencing 
quality of this fish. This hypothesis is supported by the fact that two-thirds of the reads from this individual were discarded during multiplexing for this dataset. It is worth noting that the peak memory usage and most of the runtime were in the process command, which needs to be run only once for each dataset because filtering parameters (i.e., minimum depth for marker presence) can be applied in the later steps of the RADSex pipeline.

\section{RADSex validation on a published dataset}

To assess whether RADSex can recover previous results on sex determination, we analyzed a dataset that has been used to characterize a major sex-determination locus (Wilson et al., 2014). This dataset consists of RAD-Seq reads from 31 males and 30 females from the carbio strain of Oryzias latipes, the Japanese medaka, which has a known $\mathrm{XX/XY}$ sex determination system with chromosome 1 as the sex chromosome (Matsuda et al., 2002; Nanda et al., 2002). We analyzed this O. latipes dataset with RADSex using a minimum depth of 10 (-d 10) to define the presence of a marker in an individual. This value of minimum depth was chosen based on the median sequencing depth in each individual computed with radsex depth: the average median sequencing depth was 34 , and thus a stringent minimum depth of 10 can be used to discard markers from potential sequencing errors but still retain real markers. In total, we found 121,492 markers present in at least one individual with a minimum depth of 10, among which 194 markers were significantly associated with male phenotype (radsex signif, $p<0.05$, chi-squared test with Bonferroni correction, highlighted tiles in Fig. 4.A). Among these 194 markers, 165 were present in at least 20 of the 31 males. In addition, a single marker present in 26 of the 30 females and 5 males was significantly associated with female phenotype (Fig. 4.A). However, we did not find any marker simultaneously present in more than 29 of the 31 phenotypic males and absent from all phenotypic females. To understand why no marker was found exclusively in all males given that medaka has an XY/XX sex determination system, we extracted individual depth for all 165 markers present in at least 20 males and none of the females using radsex subset. We clustered both markers and individuals separately based on these depth values and generated a heatmap of the results using the radsex_markers_depth() function of sgtr (Fig. 4.B). As expected, phenotypic males and females clustered in two separate groups, except for two phenotypic males (green arrows in Fig. 4.B) that clustered with the phenotypic females, showing zero or very low depth for the extracted markers. These two outlier phenotypic males had already been found to be sex-reversed genetic $\mathrm{XX}$ females by retrospective genotyping in Wilson et al. 
(Wilson et al., 2014). After correcting the designated phenotypic sex of these two individuals, radsex signif yielded 232 markers significantly associated with male phenotype and no marker significantly associated with female phenotype ( $p<0.05$, chi-squared test with Bonferroni correction), as expected for an $X Y / X X$ system. In addition, six males forming a sub-cluster within the male cluster from sgtr radsex_markers_depth() had low or null depth for almost half of the extracted markers, suggesting that several $\mathrm{Y}$ chromosome haplotypes could be present in the sequenced population.

To identify the sex chromosome and to delimit the sex-differentiated region, we aligned the RADSex markers to the assembly used by Wilson et al. (MEDAKA1, available at http://77.235.253.122:8012/Oryzias_latipes/Info/Index) using radsex map. Among the 232 markers significantly associated with male phenotype after reassignment of the sex-reversed fish, 131 (56\%) aligned to LG01 of O. latipes (Ola1), three (1\%) aligned to other chromosomes, $54(23 \%)$ aligned to unplaced scaffolds, and 44 (19\%) were not uniquely aligned with mapping quality higher than 20 (Fig. 4.C). On Ola1, markers significantly associated with male phenotype aligned to a continuous region spanning from 14.7 $\mathrm{Mb}$ to $31.9 \mathrm{Mb}$ (Fig. 4.D). Within this region, markers found in all males and no females aligned between 17 and $23 \mathrm{Mb}$ (green box in Fig. 4.D) and markers present in all but the six males identified in the clustering step and absent from all females aligned between 14.7 Mb and $31.9 \mathrm{Mb}$ (purple boxes in Fig. 4.D). This result supports the hypothesis that multiple $Y$ chromosome haplotypes are present in this medaka population, with one region located between 17 and $23 \mathrm{Mb}$ on Ola1 showing strong $\mathrm{X} / \mathrm{Y}$ differentiation in all $\mathrm{Y}$ haplotypes, and a wider region located between 14.7 and $31.9 \mathrm{Mb}$ on Ola1 showing strong $X / Y$ differentiation in only one $Y$ haplotype.

To evaluate whether markers created by RADSex in the medaka dataset represent biological alleles, we computed the number of markers at each alignment starting position on the genome sequence from the results of radsex map. The vast majority (94\%) of positions had one (65\%) or two $(29 \%)$ markers aligned, and less than $1 \%$ of positions had more than three markers aligned, as expected for alleles in biological loci (Supp. Fig. 2.A). The original study by Wilson et al. (2014) identified 248 SNPs associated with male phenotype aligned to a region ranging from 14.3 to 32.5 Mb on Ola1. To compare sex-biased RADSex markers with the sex-biased SNPs from Wilson et al. (2014), the genomic position and p-value of association with sex were retrieved for all 248 sexbiased SNPs from Supplementary Table S1 from Wilson et al.(2014). Among these SNPs, 153 (62\%) were located within a RADSex marker significantly associated with sex, 89 (36\%) were located within a RADSex marker not significantly associated with sex, and six (2\%) were located at a position where no RADSex marker was aligned (Supp. Fig. 2.B). The discrepancy for these 89 SNPs can be 
explained by two factors. First, although p-values of association with sex for RADSex markers were correlated with p-values for SNPs from Wilson et al. (2014) aligned within a marker, p-values were generally slightly lower for Wilson et al. (2014) (Supp. Fig. 2.C). Second, the number of markers used for Bonferroni correction was higher for RADSex (121,492 markers, threshold indicated by the blue dashed line in Supp. Fig. 2.C) than for Wilson et al. (2014) ( 700 SNPs after filtering out SNPs present in $<75 \%$ males or females, threshold indicated by the red horizontal dashed line in Supp. Fig. 2C). Using the same threshold for Bonferroni correction (red vertical dashed line in Supp. Fig. 2.C), the number of SNPs located within RADSex markers not significantly associated with sex dropped to 22 SNPs (9\%). In addition, RADSex markers significantly associated with sex were aligned at 67 new positions (38\%) that did not contain any sex-biased SNPs from Wilson et al. (Supp. Fig. 2.D), 40 of which (60\%) were aligned to Ola1 between $14.6 \mathrm{Mb}$ and $32.5 \mathrm{Mb}$.

Overall, these results indicate that markers identified as significantly associated by RADSex are consistent with sex-biased SNPs from Wilson et al. (Wilson et al., 2014), but the threshold for association with sex is more stringent in RADSex. Despite this increased stringency, RADSex identified markers significantly associated with sex for 67 genomic locations that were not identified in the original study. Furthermore, our analysis revealed a previously unidentified Y-specific polymorphism in the sequenced population. This example highlights the effectiveness and versatility of RADSex and its visualization tools to identify, explore, and explain patterns in datasets that were previously overlooked.

\section{Analysis of 15 new ray-finned fish RAD-sequencing datasets}

RAD-Seq datasets were generated for 15 ray-finned species in which no markers for genetic sex determination were available when we initiated this work. For each species, we used RADSex to search for sex-biased markers with a minimum marker depth (-d) of 1, 2, 5, and 10. We report results for a minimum marker depth of 10 (Table 1) because it is the most stringent, except for two species that had the lowest sequencing depths, for which we report results for a minimum marker depth of 2 (Supp. Fig. 3). We identified an $X X / X Y$ sex determining system in six species (Cyprinus carpio, Gymnotus carapo, Plecoglossus atlivelis, Tinca tinca, Gadus morhua, and Poecilia sphenops) with a few male and/or female outliers in four of these species (Fig. 5). Markers significantly associated with phenotypic sex for each species are provided in Supp. Table 6. An XX/XY SD system is in agreement with previous reports for C. carpio (Gomelsky, 2003), G. carapo (da Silva, Matoso, Artoni, 
\& Feldberg, 2014), P. altivelis (Watanabe, Yamasaki, Seki, \& Taniguchi, 2004), and G. morhua (Haugen et al., 2012; Whitehead, Benfey, \& Martin-Robichaud, 2012). The XX/XY SD system inferred from RADSex results for $P$. sphenops conflicts with published findings on an ornamental population for that species that showed a female heterogametic system (Nanda, Schartl, Epplen, Feichtinger, \& Schmid, 1993). However, alternative population-specific sex determination systems have also been described for $P$. sphenops (Volff \& Schartl, 2001). Because these studies were performed on ornamental fish and laboratory strains, previously derived conclusions may not apply to wild populations like the one we analyzed. Lastly, to the best of our knowledge, sex determination was not previously characterized in the tench, Tinca tinca.

In G. morhua, three markers were significantly associated with male phenotype, but they were also found in two females and none of the three markers was present in more than 27 of the 34 males (Fig. 5.B). Furthermore, multiple markers were found predominantly in males but not significantly associated with sex, with eight markers consistently present with depth higher than five in 20 males and absent from all females (these markers are highlighted with arrows in Fig. 6.A). The three markers significantly associated with sex (red-outlined boxes) and most other markers strongly but not significantly associated with sex aligned to cod linkage group 11 (Gmo11) (Fig. 6.B and 6.C). Markers strongly associated with sex aligned to a region spanning from 0 to $20 \mathrm{Mb}$ on $\mathrm{Gmo11}$, and the three markers significantly associated with sex aligned between 10 to $15 \mathrm{Mb}$ on this chromosome in a region that was previously characterized as containing the sex locus in this species (Kirubakaran et al., 2019; Star et al., 2016). These results confirm the previously identified XX/XY sex determining system and Gmo11 as the sex chromosome in Atlantic cod (Haugen et al., 2012; Whitehead et al., 2012), but our findings suggest an additional sex determination complexity in the studied Atlantic cod aquaculture population that had not been observed before in wild populations of Atlantic cod (Kirubakaran et al., 2019; Star et al., 2016). The incomplete sex-linkage of sex-biased markers may be explained by a Y-chromosome polymorphism and/or the existence of sex-reversed genetic $\mathrm{XX}$ females. Although $\mathrm{Y}$-chromosome population differences have rarely been explored, at least in fish species, the results from our re-analysis of a medaka population suggest that such polymorphisms may be more frequent than expected. In addition, female-to-male sex-reversal could be a consequence of intensive aquaculture rearing conditions leading to stress-induced sex-reversal (Geffroy \& Douhard, 2019), which has been observed in other fish species reared in laboratory or aquaculture facilities (Pan et al., 2019). 
In the nine other species (Table 1 and Supp. Fig. 4), we did not find any marker significantly associated with phenotypic sex, which indicates that these species either 1) have a small undifferentiated sex locus, 2) lack a genetic sex determination mechanism, or 3) rely on a complex combination of environmental and genetic sex determination factors that prevented the detection of a clear sex-biased signal in the dataset.

\section{DISCUSSION}

We demonstrate that RADSex is an efficient and -- thanks to its simple usage and visualization options -- user-friendly tool to identify sex-determination systems and sex-biased markers from RAD-Seq data. Unlike other popular RAD-Seq analysis software, e.g., Stacks (Catchen et al., 2011) and PyRad (Eaton, 2014), which catalog polymorphic loci, RADSex creates monomorphic markers. RADSex therefore counts as a separate marker each allele at a locus defined as polymorphic by other software. Working with monomorphic markers allows statistical comparison of populations using straightforward presence/absence tests, yielding results that are comparable between datasets. In contrast, the outcome of grouping sequences into polymorphic markers can be sensitive to parameter values (Paris et al., 2017; Rodríguez-Ezpeleta et al., 2016; Shafer et al., 2018). Leveraging this property of monomorphic markers, RADSex offers a simple and reproducible way to identify de novo genetic differences between females and males and to locate these differences on a genome when a reference assembly is available. In addition to this simplicity, RADSex requires few resources and provides comprehensive visualization tools to assist the interpretation of its output.

In particular, the tile plot visualization provides a comprehensive overview of the RAD-Seq results across all individuals and allows the user to quickly identify straightforward cases of genetic sex determination systems. For instance, $X X / X Y$ or ZZ/ZW monofactorial systems can be easily detected in absence of any major environmental effects on sex, as demonstrated by our analyses on the ayu ( $P$. altivelis) and the tench ( $T$. tinca) datasets: in both species, the tile plot revealed complete sex-linkage of several markers significantly associated with male phenotype, indicating a male heterogametic sex determination system. Besides these straightforward cases, the tile plot visualization is useful to identify the sex-determination system in more complex datasets, for instance in the presence of outliers and sequencing biases. These complex datasets can then be further explored with the marker depths heatmap visualization, for instance to confirm whether sex-biased 
markers are absent or present in specific individuals. Using this heatmap, we were able to quickly identify sex-reversed individuals responsible for the non-complete sex-linkage of sex-biased markers in a publicly available medaka dataset (Wilson et al., 2014), as well as outliers in our own common carp (C. carpio), Atlantic cod (G. morhua), banded knifefish (G. carapo), and common molly ( $P$. sphenops) datasets. These outliers could arise from human errors in assessing phenotypic sex; in particular, in the common molly, males are externally indistinguishable from females until puberty, which occurs late in some males, and immature testes resemble non-reproductively active ovaries. Because in this species sex was determined from secondary sex characters and macroscopy of the gonads, but not histology, it is possible that the relatively high number of females in which we found male-biased markers were immature males that were mis-sexed as females. The presence of outliers, however, could also hint at a more complex sex determination system, for instance polygenic sex-determination (Moore \& Roberts, 2013), autosomal modifiers that are polymorphic in the population $(\mathrm{Wu}, 1983)$, or simple monofactorial genetic sex determination systems with some sex-reversed individuals triggered by environmental factors (Baroiller \& D'Cotta, 2016; Dupoué et al., 2019; Wessels et al., 2017). With a high frequency of outliers, sex-biased markers may no longer be significantly associated with phenotypic sex, yet these markers can still be biologically relevant.

Because RADSex creates non-polymorphic markers, the number and distribution of markers between individuals is only affected by a single parameter controlling the minimum depth to consider a marker present or absent in an individual. An overly high minimum depth value can lead to false negatives, i.e., markers considered absent from an individual because of insufficient sequencing depth, and a very low minimum depth value can create false positives, i.e., markers considered present in an individual because of sequencing errors. Moreover, because the probability of association with phenotypic sex for a marker is adjusted using Bonferroni correction, the stringency of the test for significance increases with the total number of markers identified in the dataset, which is affected by the minimum depth parameter. In the 15 datasets that we generated, median sequencing coverage in an individual was between 30x and 100x, except for three species for which it was lower than 30x (Supp. Fig. 3). For all species except the three species with low sequencing coverage, we found that minimum depth values between 5 and 10 provided a good balance between stringency and minimizing false negatives. Some datasets, however, may require using low minimum depth values, for instance the three datasets for which median individual sequencing depth was low, or species with large genome sizes like some sharks (Hara et al., 2018) or some amphibians (Sclavi \& Herrick, 2019) for which sequencing all individuals of a population with sufficient depth can be 
costly. In such cases, the memory efficiency of RADSex enables the analysis of large datasets with minimum depth as low as one, which can require a lot of memory with other software. In addition to sequencing errors, common sources of biases in RAD-Sequencing analyses include highly repeated sequences (Catchen et al., 2011), PCR duplicates, and allele drop-out (Andrews et al., 2016). RADSex considers presence and absence of markers and highly repeated sequences are treated like any other, meaning that RADSex is able to identify sex-biased repeated sequences. PCR duplicates can produce false negatives if one allele at a heterozygous locus is under-amplified in one individual, resulting in a sequencing depth lower than the minimum depth threshold for this allele in this individual; this effect can be mitigated by using a low minimum depth threshold. Allele drop-out would also generate false positives and cannot be avoided with RADSex; however, the visualization tools included in RADSex help identifying scenarios where the sex signal is tempered by false negatives.

The numbers of markers significantly associated with phenotypic sex can provide an estimate of the size of the sex determining region, with a low or high number of sex-specific markers reflecting a small or a large non-recombining region, respectively. It is worth noting, however, that other factors can affect this number, for instance the level of differentiation between males and females within the non-recombining region, or bias in GC content within this region (Sigeman et al., 2018; Smeds et al., 2015) potentially leading to an over- or under-representation of RAD-Seq markers depending on the restriction enzyme used for library construction. An extreme case is a complete absence of markers associated with phenotypic sex for any value of minimum depth, which we observed in nine of our datasets. These null results may be linked to two key features of sex determination in teleosts. First, early cytological studies have shown that the majority of teleost fish carry homomorphic sex chromosome pairs (Devlin \& Nagahama, 2002), and more recent genomic analyses have revealed that small sex loci are common in teleosts, with the extreme case of a single SNP being the sole difference between the sexes in Fugu (Kamiya et al., 2012). In such cases with very little differentiation between the sex chromosomes, the fragmented resolution of RAD-Seq means that the likelihood of obtaining RAD sequences from the sex locus can be very low. Although this problem might be overcome by increasing the number of RAD-seq reads, for example by using a restriction enzyme that cuts more frequently, genome-wide approaches like pooled sequencing of males and females (Feron et al., 2020; Gammerdinger, Conte, Baroiller, D’Cotta, \& Kocher, 2016; Wen et al., 2019) or individual whole genome sequencing (Star et al., 2016) may be necessary to identify the sex-determining region. Second, a complete absence of sex-biased markers can reflect a complex 
sex determination system, for instance 1) a polygenic system, 2) strong polymorphism on the sex chromosomes leading to multiple $\mathrm{W}$ or $\mathrm{Y}$ haplotypes each shared by only a fraction of individuals from the heterogametic sex, or 3) a system involving a strong environmental effect that would weaken the association between genetic markers and phenotypic sex. In fish, many environmental components can interfere with genetic sex determination mechanisms (Kikuchi and Hamakuchi 2013, Heule et al., 2014). The visualization tools included in RADSex facilitate the identification of sex-reversed individuals and sex-chromosome polymorphism as demonstrated in our re-analysis of the Medaka dataset. The association between phenotypic sex and genomic regions, however, may still be difficult to detect when sex-reversal is frequent, especially when associated with phenotyping uncertainty. For such systems, pooled sequencing may not be well-suited because male and female pools will each contain heterogeneous genotypes, and individual sequencing remains expensive despite decreasing sequencing costs. A hybrid strategy using both RAD-Seq and pooled sequencing may prove to be the most efficient for these complex sex-determination systems, as demonstrated in the case of the goldfish (Wen et al., 2019), a species with a strong thermal effect on sex determination (Goto-Kazeto et al., 2006). Finally, in some species, sex is determined entirely by environmental factors and therefore does not involve any genetic mechanism (Martínez-Juárez \& Moreno-Mendoza, 2019).

One of the strengths of RAD-Seq is the ability to compare populations without a reference genome, which may not always be available for non-model species. When a genome is available, however, locating sex-biased markers provides information on the size and level of differentiation of the sex-determining region and can help to identify candidate genes involved in sex determination. The manhattan plot, circos plot, and contig plot visualizations included in RADSex quickly display the location of the sex-biased markers over the whole genome or over selected regions to identify the sex-determining region. Furthermore, the sex-bias metric and probability of association with sex computed with RADSex can be more effective than $F_{S T}$ computed on polymorphic loci to detect variability in the sex-determining region. This was illustrated by our reanalysis of a publicly available Japanese medaka dataset (Wilson et al., 2014), in which we detected multiple $Y$ chromosome haplotypes that were not found in the original study. Intra-specific sex chromosome polymorphisms have been reported in different taxa, including mammals in which $\mathrm{Y}$ chromosome polymorphisms are widely used for human evolution studies (Jobling \& Tyler-Smith, 2003) and some fruit flies in which the $\mathrm{Y}$ chromosome polymorphism is thought to control male fitness (Chippindale \& Rice, 2001). In fish, sex chromosome polymorphisms have been reported in different populations of the guppy, 
Poecilia reticulata, and in other closely related species of guppies (Nanda et al., 2014), and different sex chromosomes have even been found within the same species, for instance in tilapias (Cnaani et al., 2008). To the best of our knowledge, however, there are no reports of intra-specific population differences of a single sex chromosome in fish from genomic data, and our results provide the first example of such a $\mathrm{Y}$ chromosome polymorphism in this clade.

As part of this study, we used RADSex to investigate the sex determination systems of 15 species sampled broadly across the ray-finned fish phylogeny. We identified XX/XY SD systems in six of these 15 species but did not find evidence of a ZZ/ZW SD system in any of the other nine species. Although our sampling is too limited to make general inferences about teleost sex determination, the predominance of XX/XY over ZZ/ZW SD systems in our datasets is in agreement with previous findings that in teleosts, transitions from ZZ/ZW to XX/XY SD systems are more frequent than the reverse (Pennell, Mank, \& Peichel, 2018), which would result in XY SD systems being prevalent in this clade.

All of our datasets were generated using single-digest RAD-Seq, but the workflow accepts double-digest RAD-Seq data as well. Furthermore, although all species included in this study are fish, we did not use any fish-specific assumptions in RADSex's implementation, and therefore the computational workflow can be applied to RAD-Seq data from any species. Finally, we specifically developed RADSex to study sex determination, and this design decision is reflected in the wording of this manuscript. However, the computational workflow was designed to be generic, and therefore both the radsex software and the sgtr $\mathrm{R}$ package could be used for other major quantitative trait loci with contrasting binary phenotypes, whether naturally occurring or induced by mutagenesis.

\section{ACKNOWLEDGEMENTS}

We thank all colleagues who helped us improve the beta version of RADSex by testing it and providing feedback. We thank Daniel Jeffries for his feedback on the software and enriching discussions on our results. This project was supported by funds from the "Agence Nationale de la Recherche", the "Deutsche Forschungsgemeinschaft" (ANR/DFG, PhyloSex project, 2014-2016, SCHA 408/10-1, MS), the National Institutes of Health (USA) grants R01GM085318 and R35GM139635, JHP), and the National Science Foundation (USA) Office of Polar Programs (grants PLR-1247510 and PLR-1444167 to HWD and grant OPP-1543383 to JHP, TD, and HWD). The MGX core sequencing facility was supported by France Genomique National infrastructure, funded as part 
of "Investissement d'avenir" program managed by Agence Nationale pour la Recherche (contract ANR-10-INBS-09). RF was partially supported by Swiss National Science Foundation grant PP00P3_170664 to RMW. Common carp were provided by the PEARL INRA 1036 U3E experimental facilities that are supported by the ANAEE-France National Infrastructure. We thank Allyse Ferrara, Quenton Fontenot, and the Bayousphere Lab at Nicholls State University (Thibodeaux, LA) for support with spotted gar sample collection and preparation, Dan Rosauer and Jonathan Meerbeek from the lowa Department of Natural Resources for walleye sampling. The authors thank the captain and crew of the ARSV Laurence M. Gould and the personnel of the US Antarctic Support Contractor for assistance in Chile, at sea, at Palmer Station, Antarctica, and logistically in Denver, CO. Part of this work was supported by a contribution from the Marine Science Center at Northeastern University (contribution \#TBD). We are grateful to the genotoul bioinformatics platform Toulouse Occitanie (Bioinfo Genotoul, doi: 10.15454/1.5572369328961167E12) for providing computing and storage resources.

\section{REFERENCES}

Al-Ameri, A. A., Al-Qurainy, F., Gaafar, A.-R. Z., Khan, S., \& Nadeem, M. (2016). Molecular Identification of Sex in Phoenix dactylifera Using Inter Simple Sequence Repeat Markers. BioMed Research International, 2016. doi: 10.1155/2016/4530846

Amores, A., Catchen, J., Ferrara, A., Fontenot, Q., \& Postlethwait, J. H. (2011). Genome evolution and meiotic maps by massively parallel DNA sequencing: Spotted gar, an outgroup for the teleost genome duplication. Genetics, 188(4), 799-808. doi: 10.1534/genetics.111.127324

Ancona, S., Dénes, F. V., Krüger, O., Székely, T., \& Beissinger, S. R. (2017). Estimating adult sex ratios in nature. Philosophical Transactions of the Royal Society of London. Series B, Biological Sciences, 372(1729). doi: 10.1098/rstb.2016.0313

Andrews, K. R., Good, J. M., Miller, M. R., Luikart, G., \& Hohenlohe, P. A. (2016). Harnessing the power of RADseq for ecological and evolutionary genomics. Nature Reviews. Genetics, 17(2), 81-92. doi: 10.1038/nrg.2015.28

Bachtrog, D. (2008). The temporal dynamics of processes underlying Y chromosome degeneration. Genetics, 179(3), 1513-1525. doi: 10.1534/genetics.107.084012

Bachtrog, D., Mank, J. E., Peichel, C. L., Kirkpatrick, M., Otto, S. P., Ashman, T.-L., ... Consortium, 
T. T. of S. (2014). Sex Determination: Why So Many Ways of Doing It? PLOS Biology, 12(7), e1001899. doi: 10.1371/journal.pbio.1001899

Baroiller, J.-F., \& D'Cotta, H. (2016). The Reversible Sex of Gonochoristic Fish: Insights and Consequences. Sexual Development: Genetics, Molecular Biology, Evolution, Endocrinology, Embryology, and Pathology of Sex Determination and Differentiation, 10(5-6), 242-266. doi: $10.1159 / 000452362$

Benestan, L., Moore, J.-S., Sutherland, B. J. G., Le Luyer, J., Maaroufi, H., Rougeux, C., ... Bernatchez, L. (2017). Sex matters in massive parallel sequencing: Evidence for biases in genetic parameter estimation and investigation of sex determination systems. Molecular Ecology, 26(24), 6767-6783. doi: 10.1111/mec.14217

Bewick, A. J., Chain, F. J. J., Zimmerman, L. B., Sesay, A., Gilchrist, M. J., Owens, N. D. L., ... Evans, B. J. (2013). A large pseudoautosomal region on the sex chromosomes of the frog Silurana tropicalis. Genome Biology and Evolution, 5(6), 1087-1098. doi: 10.1093/gbe/evt073 Blackmon, H., Ross, L., \& Bachtrog, D. (2017). Sex Determination, Sex Chromosomes, and Karyotype Evolution in Insects. The Journal of Heredity, 108(1), 78-93. doi: 10.1093/jhered/esw047

Carmichael, S. N., Bekaert, M., Taggart, J. B., Christie, H. R. L., Bassett, D. I., Bron, J. E., ... Sturm, A. (2013). Identification of a sex-linked SNP marker in the salmon louse (Lepeophtheirus salmonis) using RAD sequencing. PloS One, 8(10), e77832. doi:

10.1371/journal.pone.0077832

Catchen, J., Hohenlohe, P. A., Bassham, S., Amores, A., \& Cresko, W. A. (2013). Stacks: An analysis tool set for population genomics. Molecular Ecology, 22(11), 3124-3140. doi: 10.1111/mec. 12354

Catchen, J. M., Amores, A., Hohenlohe, P., Cresko, W., \& Postlethwait, J. H. (2011). Stacks: Building and genotyping Loci de novo from short-read sequences. G3 (Bethesda, Md.), 1(3), 171-182. doi: $10.1534 / \mathrm{g} 3.111 .000240$

Charlesworth, B., \& Charlesworth, D. (2000). The degeneration of Y chromosomes. Philosophical Transactions of the Royal Society of London. Series B, Biological Sciences, 355(1403), 15631572. doi: 10.1098/rstb.2000.0717 
Chippindale, A. K., \& Rice, W. R. (2001). Y chromosome polymorphism is a strong determinant of male fitness in Drosophila melanogaster. Proceedings of the National Academy of Sciences of the United States of America, 98(10), 5677-5682. doi: 10.1073/pnas.101456898

Cnaani, A., Lee, B.-Y., Zilberman, N., Ozouf-Costaz, C., Hulata, G., Ron, M., ... Kocher, T. D. (2008). Genetics of sex determination in tilapiine species. Sexual Development: Genetics, Molecular Biology, Evolution, Endocrinology, Embryology, and Pathology of Sex Determination and Differentiation, 2(1), 43-54. doi: 10.1159/000117718

Corcoran, P., Anderson, J. L., Jacobson, D. J., Sun, Y., Ni, P., Lascoux, M., \& Johannesson, H. (2016). Introgression maintains the genetic integrity of the mating-type determining chromosome of the fungus Neurospora tetrasperma. Genome Research. doi: 10.1101/gr.197244.115

da Silva, M., Matoso, D. A., Artoni, R. F., \& Feldberg, E. (2014). New approach data in electric fish (Teleostei: Gymnotus): sex chromosome evolution and repetitive DNA. Zebrafish, 11(6), 528535. doi: 10.1089/zeb.2013.0966

Dan, C., Mei, J., Wang, D., \& Gui, J.-F. (2013). Genetic Differentiation and Efficient Sex-specific Marker Development of a Pair of Y - and X-linked Markers in Yellow Catfish. International Journal of Biological Sciences, 9(10), 1043-1049. doi: 10.7150/ijbs.7203

Davey, J. W., Hohenlohe, P. A., Etter, P. D., Boone, J. Q., Catchen, J. M., \& Blaxter, M. L. (2011). Genome-wide genetic marker discovery and genotyping using next-generation sequencing. Nature Reviews. Genetics, 12(7), 499-510. doi: 10.1038/nrg3012

David, C. J., \& Pandian, T. J. (2016). A RAPID PCR ASSAY FOR IDENTIFICATION OF MALES IN WIDOW TETRA, GYMNOCORYMBUS TERNETZI (BOULENGER, 1895). European Journal of Biomedical, 3(6), 436-440.

Devlin, R. H., \& Nagahama, Y. (2002). Sex determination and sex differentiation in fish: An overview of genetic, physiological, and environmental influences. Aquaculture, 208(3), 191364. doi: 10.1016/S0044-8486(02)00057-1

Doorn, G. S. van, \& Kirkpatrick, M. (2007). Turnover of sex chromosomes induced by sexual conflict. Nature, 449(7164), 909-912. doi: 10.1038/nature06178

Drinan, D. P., Loher, T., \& Hauser, L. (2018). Identification of Genomic Regions Associated With 
Sex in Pacific Halibut. The Journal of Heredity, 109(3), 326-332. doi: 10.1093/jhered/esx 102 Dupoué, A., Lourdais, O., Meylan, S., Brischoux, F., Angelier, F., Rozen-Rechels, D., ... Le Galliard, J.-F. (2019). Some like it dry: Water restriction overrides heterogametic sex determination in two reptiles. Ecology and Evolution, 9(11), 6524-6533. doi: 10.1002/ece3.5229

Eaton, D. A. R. (2014). PyRAD: Assembly of de novo RADseq loci for phylogenetic analyses. Bioinformatics (Oxford, England), 30(13), 1844-1849. doi: 10.1093/bioinformatics/btu121

Feron, R., Zahm, M., Cabau, C., Klopp, C., Roques, C., Bouchez, O., ... Guiguen, Y. (2020). Characterization of a Y-specific duplication/insertion of the anti-Mullerian hormone type II receptor gene based on a chromosome-scale genome assembly of yellow perch, Perca flavescens. Molecular Ecology Resources, 20(2), 531-543. doi: 10.1111/1755-0998.13133

Gamble, T. (2016). Using RAD-seq to recognize sex-specific markers and sex chromosome systems. Molecular Ecology, 25(10), 2114-2116. doi: 10.1111/mec.13648

Gamble, T., Castoe, T. A., Nielsen, S. V., Banks, J. L., Card, D. C., Schield, D. R., ... Booth, W. (2017). The Discovery of XY Sex Chromosomes in a Boa and Python. Current Biology: $C B$, 27(14), 2148-2153.e4. doi: 10.1016/j.cub.2017.06.010

Gamble, T., Coryell, J., Ezaz, T., Lynch, J., Scantlebury, D. P., \& Zarkower, D. (2015). Restriction Site-Associated DNA Sequencing (RAD-seq) Reveals an Extraordinary Number of Transitions among Gecko Sex-Determining Systems. Molecular Biology and Evolution, 32(5), 1296-1309. doi: 10.1093/molbev/msv023

Gamble, T., McKenna, E., Meyer, W., Nielsen, S. V., Pinto, B. J., Scantlebury, D. P., \& Higham, T. E. (2018). XX/XY Sex Chromosomes in the South American Dwarf Gecko (Gonatodes humeralis). The Journal of Heredity, 109(4), 462-468. doi: 10.1093/jhered/esx112

Gamble, T., \& Zarkower, D. (2014). Identification of sex-specific molecular markers using restriction site-associated DNA sequencing. Molecular Ecology Resources, 14(5), 902-913. doi: 10.1111/1755-0998.12237

Gammerdinger, W. J., Conte, M. A., Baroiller, J.-F., D’Cotta, H., \& Kocher, T. D. (2016). Comparative analysis of a sex chromosome from the blackchin tilapia, Sarotherodon melanotheron. BMC Genomics, 17(1), 808. doi: 10.1186/s12864-016-3163-7

Geffroy, B., \& Douhard, M. (2019). The Adaptive Sex in Stressful Environments. Trends in Ecology 
\& Evolution, 34(7), 628-640. doi: 10.1016/j.tree.2019.02.012

Gomelsky, B. (2003). Chromosome set manipulation and sex control in common carp: A review. Aquatic Living Resources, 16(5), 408-415. doi: 10.1016/S0990-7440(03)00085-8

Goodenough, U., \& Heitman, J. (2014). Origins of eukaryotic sexual reproduction. Cold Spring Harbor Perspectives in Biology, 6(3). doi: 10.1101/cshperspect.a016154

Goto-Kazeto, R., Abe, Y., Masai, K., Yamaha, E., Adachi, S., \& Yamauchi, K. (2006). Temperaturedependent sex differentiation in goldfish: Establishing the temperature-sensitive period and effect of constant and fluctuating water temperatures. Aquaculture, 254(1), 617-624. doi: 10.1016/j.aquaculture.2005.10.009

Gu, L., Walters, J. R., \& Knipple, D. C. (2017). Conserved Patterns of Sex Chromosome Dosage Compensation in the Lepidoptera (WZ/ZZ): Insights from a Moth Neo-Z Chromosome. Genome Biology and Evolution, 9(3), 802-816. doi: 10.1093/gbe/evx039

Gu, Z., Gu, L., Eils, R., Schlesner, M., \& Brors, B. (2014). Circlize implements and enhances circular visualization in R. Bioinformatics, 30(19), 2811-2812. doi: 10.1093/bioinformatics/btu393

Hara, Y., Yamaguchi, K., Onimaru, K., Kadota, M., Koyanagi, M., Keeley, S. D., .. Kuraku, S. (2018). Shark genomes provide insights into elasmobranch evolution and the origin of vertebrates. Nature Ecology \& Evolution, 2(11), 1761-1771. doi: 10.1038/s41559-018-0673-5

Haugen, T., Almeida, F. F., Andersson, E., Bogerd, J., Male, R., Skaar, K. S., ... Taranger, G. L. (2012). Sex differentiation in Atlantic cod (Gadus morhua L.): Morphological and gene expression studies. Reproductive Biology and Endocrinology: $R B \& E, 10,47$. doi: $10.1186 / 1477-7827-10-47$

Hohenlohe, P. A., Catchen, J., \& Cresko, W. A. (2012). Population genomic analysis of model and nonmodel organisms using sequenced RAD tags. Methods in Molecular Biology (Clifton, N.J.), 888, 235-260. doi: 10.1007/978-1-61779-870-2_14

Huylmans, A. K., Macon, A., \& Vicoso, B. (2017). Global Dosage Compensation Is Ubiquitous in Lepidoptera, but Counteracted by the Masculinization of the Z Chromosome. Molecular Biology and Evolution, 34(10), 2637-2649. doi: 10.1093/molbev/msx 190

Jobling, M. A., \& Tyler-Smith, C. (2003). The human Y chromosome: An evolutionary marker comes of age. Nature Reviews Genetics, 4(8), 598-612. doi: 10.1038/nrg1124 
Kafkas, S., Khodaeiaminjan, M., Güney, M., \& Kafkas, E. (2015). Identification of sex-linked SNP markers using RAD sequencing suggests $\mathrm{ZW} / \mathrm{ZZ}$ sex determination in Pistacia vera L. BMC Genomics, 16, 98. doi: 10.1186/s12864-015-1326-6

Kamiya, T., Kai, W., Tasumi, S., Oka, A., Matsunaga, T., Mizuno, N., ... Kikuchi, K. (2012). A trans-species missense SNP in Amhr2 is associated with sex determination in the tiger pufferfish, Takifugu rubripes (fugu). PLoS Genetics, 8(7), e1002798. doi: 10.1371/journal.pgen.1002798

Karlsen, Ø., Meeren, T. van der, Rønnestad, I., Mangor-Jensen, A., Galloway, T. F., Kjørsvik, E., \& Hamre, K. (2015). Copepods enhance nutritional status, growth and development in Atlantic cod (Gadus morhua L.) larvae - Can we identify the underlying factors? PeerJ, 3, e902. doi: 10.7717 peerj. 902

Kikuchi, K., \& Hamaguchi, S. (2013). Novel sex-determining genes in fish and sex chromosome evolution. Developmental Dynamics: An Official Publication of the American Association of Anatomists, 242(4), 339-353. doi: 10.1002/dvdy.23927

Kirubakaran, T. G., Andersen, Ø., De Rosa, M. C., Andersstuen, T., Hallan, K., Kent, M. P., \& Lien, S. (2019). Characterization of a male specific region containing a candidate sex determining gene in Atlantic cod. Scientific Reports, 9(1), 116. doi: 10.1038/s41598-018-36748-8

Li, H. (2013). Aligning sequence reads, clone sequences and assembly contigs with BWA-MEM. ArXiv:1303.3997 [q-Bio]. Retrieved from http://arxiv.org/abs/1303.3997

Li, H., Handsaker, B., Wysoker, A., Fennell, T., Ruan, J., Homer, N., ... 1000 Genome Project Data Processing Subgroup. (2009). The Sequence Alignment/Map format and SAMtools. Bioinformatics (Oxford, England), 25(16), 2078-2079. doi: 10.1093/bioinformatics/btp352

Liao, Z., Yu, Q., \& Ming, R. (2017). Development of male-specific markers and identification of sex reversal mutants in papaya. Euphytica, 213(2), 53. doi: 10.1007/s10681-016-1806-z

Martínez-Juárez, A., \& Moreno-Mendoza, N. (2019). Mechanisms related to sexual determination by temperature in reptiles. Journal of Thermal Biology, 85, 102400. doi:

10.1016/j.jtherbio.2019.102400

Mathers, T. C., Hammond, R. L., Jenner, R. A., Hänfling, B., Atkins, J., \& Gómez, A. (2015). Transition in sexual system and sex chromosome evolution in the tadpole shrimp Triops 
cancriformis. Heredity, 115(1), 37-46. doi: 10.1038/hdy.2015.10

Matsuda, M., Nagahama, Y., Shinomiya, A., Sato, T., Matsuda, C., Kobayashi, T., ... Sakaizumi, M. (2002). DMY is a Y-specific DM-domain gene required for male development in the medaka fish. Nature, 417(6888), 559-563. doi: 10.1038/nature751

Miura, I. (2017). Sex Determination and Sex Chromosomes in Amphibia. Sexual Development: Genetics, Molecular Biology, Evolution, Endocrinology, Embryology, and Pathology of Sex Determination and Differentiation, 11(5-6), 298-306. doi: 10.1159/000485270

Modi, W. S., \& Crews, D. (2005). Sex chromosomes and sex determination in reptiles. Current Opinion in Genetics \& Development, 15(6), 660-665. doi: 10.1016/j.gde.2005.09.009

Mölder F, Jablonski KP, Letcher B, ... Köster, J. (2021) Sustainable data analysis with Snakemake [version 1; peer review: awaiting peer review]. F1000Research. 10:33. doi:10.12688/f1000research.29032.1

Moore, E. C., \& Roberts, R. B. (2013). Polygenic sex determination. Current Biology: CB, 23(12), R510-512. doi: 10.1016/j.cub.2013.04.004

Muyle, A., Zemp, N., Deschamps, C., Mousset, S., Widmer, A., \& Marais, G. A. B. (2012). Rapid De Novo Evolution of X Chromosome Dosage Compensation in Silene latifolia, a Plant with Young Sex Chromosomes. PLOS Biology, 10(4), e1001308. doi:

10.1371/journal.pbio. 1001308

Nanda, I., Schartl, M., Epplen, J. T., Feichtinger, W., \& Schmid, M. (1993). Primitive sex chromosomes in poeciliid fishes harbor simple repetitive DNA sequences. The Journal of Experimental Zoology, 265(3), 301-308. doi: 10.1002/jez.1402650311

Nanda, Indrajit, Kondo, M., Hornung, U., Asakawa, S., Winkler, C., Shimizu, A., ... Schartl, M. (2002). A duplicated copy of DMRT1 in the sex-determining region of the $\mathrm{Y}$ chromosome of the medaka, Oryzias latipes. Proceedings of the National Academy of Sciences of the United States of America, 99(18), 11778-11783. doi: 10.1073/pnas.182314699

Nanda, Indrajit, Schories, S., Tripathi, N., Dreyer, C., Haaf, T., Schmid, M., \& Schartl, M. (2014). Sex chromosome polymorphism in guppies. Chromosoma, 123(4), 373-383. doi: 10.1007/s00412-014-0455-z

Nielsen, S. V., Banks, J. L., Diaz, R. E., Trainor, P. A., \& Gamble, T. (2018). Dynamic sex 
chromosomes in Old World chameleons (Squamata: Chamaeleonidae). Journal of Evolutionary Biology, 31(4), 484-490. doi: 10.1111/jeb.13242

Nielsen, Stuart V., Daza, J. D., Pinto, B. J., \& Gamble, T. (2019). ZZ/ZW Sex Chromosomes in the Endemic Puerto Rican Leaf-Toed Gecko (Phyllodactylus wirshingi). Cytogenetic and Genome Research, 157(1-2), 89-97. doi: 10.1159/000496379

Palmer, D. H., Rogers, T. F., Dean, R., \& Wright, A. E. (2019). How to identify sex chromosomes and their turnover. Molecular Ecology, 28(21), 4709-4724. doi: https://doi.org/10.1111/mec.15245

Pan, Q., Feron, R., Yano, A., Guyomard, R., Jouanno, E., Vigouroux, E., ... Guiguen, Y. (2019). Identification of the master sex determining gene in Northern pike (Esox lucius) reveals restricted sex chromosome differentiation. PLOS Genetics, 15(8), e1008013. doi: 10.1371/journal.pgen.1008013

Paris, J. R., Stevens, J. R., \& Catchen, J. M. (2017). Lost in parameter space: A road map for stacks. Methods in Ecology and Evolution, 8(10), 1360-1373. doi: 10.1111/2041-210X.12775

Peichel, C. L., Ross, J. A., Matson, C. K., Dickson, M., Grimwood, J., Schmutz, J., ... Kingsley, D. M. (2004). The master sex-determination locus in threespine sticklebacks is on a nascent $Y$ chromosome. Current Biology: CB, 14(16), 1416-1424. doi: 10.1016/j.cub.2004.08.030

Pennell, M. W., Mank, J. E., \& Peichel, C. L. (2018). Transitions in sex determination and sex chromosomes across vertebrate species. Molecular Ecology, 27(19), 3950-3963. doi: $10.1111 / \mathrm{mec} .14540$

Pezaro, N., Doody, J. S., \& Thompson, M. B. (2017). The ecology and evolution of temperaturedependent reaction norms for sex determination in reptiles: A mechanistic conceptual model. Biological Reviews of the Cambridge Philosophical Society, 92(3), 1348-1364. doi: $10.1111 /$ brv. 12285

Piferrer, F., Blázquez, M., Navarro, L., \& González, A. (2005). Genetic, endocrine, and environmental components of sex determination and differentiation in the European sea bass (Dicentrarchus labrax L.). General and Comparative Endocrinology, 142(1-2), 102-110. doi: 10.1016/j.ygcen.2005.02.011

Pratlong, M., Haguenauer, A., Chenesseau, S., Brener, K., Mitta, G., Toulza, E., ... Pontarotti, P. 
(2017). Evidence for a genetic sex determination in Cnidaria, the Mediterranean red coral (Corallium rubrum). Royal Society Open Science, 4(3), 160880. doi: 10.1098/rsos.160880 Rochette, N. C., \& Catchen, J. M. (2017). Deriving genotypes from RAD-seq short-read data using Stacks. Nature Protocols, 12(12), 2640-2659. doi: 10.1038/nprot.2017.123

Rodríguez-Ezpeleta, N., Bradbury, I. R., Mendibil, I., Álvarez, P., Cotano, U., \& Irigoien, X. (2016). Population structure of Atlantic mackerel inferred from RAD-seq-derived SNP markers: Effects of sequence clustering parameters and hierarchical SNP selection. Molecular Ecology Resources, 16(4), 991-1001. doi: 10.1111/1755-0998.12518

Salz, H. K., \& Erickson, J. W. (2010). Sex determination in Drosophila: The view from the top. Fly, 4(1), 60-70. doi: 10.4161/fly.4.1.11277

Sclavi, B., \& Herrick, J. (2019). Genome size variation and species diversity in salamanders. Journal of Evolutionary Biology, 32(3), 278-286. doi: 10.1111/jeb.13412

Shafer, A. B. A., Peart, C. R., Tusso, S., Maayan, I., Brelsford, A., Wheat, C. W., \& Wolf, J. B. W. (2018). Bioinformatic processing of RAD-seq data dramatically impacts downstream population genetic inference. Methods in Ecology and Evolution, 907-917. doi: 10.1111/2041210X.12700@10.1111/(ISSN)2041-210x.PracticalToolsFieldMethodsMEE32018

Sigeman, H., Ponnikas, S., Videvall, E., Zhang, H., Chauhan, P., Naurin, S., \& Hansson, B. (2018). Insights into Avian Incomplete Dosage Compensation: Sex-Biased Gene Expression Coevolves with Sex Chromosome Degeneration in the Common Whitethroat. Genes, 9(8). doi: 10.3390/genes 9080373

Smeds, L., Warmuth, V., Bolivar, P., Uebbing, S., Burri, R., Suh, A., ... Ellegren, H. (2015). Evolutionary analysis of the female-specific avian W chromosome. Nature Communications, 6, 7330. doi: $10.1038 /$ ncomms 8330

Spigler, R. B., Lewers, K. S., Main, D. S., \& Ashman, T.-L. (2008). Genetic mapping of sex determination in a wild strawberry, Fragaria virginiana, reveals earliest form of sex chromosome. Heredity, 101(6), 507-517. doi: 10.1038/hdy.2008.100

Star, B., Tørresen, O. K., Nederbragt, A. J., Jakobsen, K. S., Pampoulie, C., \& Jentoft, S. (2016). Genomic characterization of the Atlantic cod sex-locus. Scientific Reports, 6, 31235. doi: $10.1038 / \operatorname{srep} 31235$ 
Utsunomia, R., Scacchetti, P. C., Hermida, M., Fernández-Cebrián, R., Taboada, X., Fernández, C., ... Martínez, P. (2017). Evolution and conservation of Characidium sex chromosomes. Heredity, 119(4), 237-244. doi: 10.1038/hdy.2017.43

Volff, J. N., \& Schartl, M. (2001). Variability of genetic sex determination in poeciliid fishes. Genetica, 111(1-3), 101-110.

Wallis, M. C., Waters, P. D., \& Graves, J. a. M. (2008). Sex determination in mammals-Before and after the evolution of SRY. Cellular and Molecular Life Sciences: CMLS, 65(20), 3182-3195. doi: 10.1007/s00018-008-8109-z

Watanabe, T., Yamasaki, K., Seki, S., \& Taniguchi, N. (2004). Detection of ayu sex-linked DNA markers using homologous clones. Fisheries Science, 70(1), 47-52. doi: 10.1111/j.14442906.2003.00769.x

Wen, M., Feron, R., Pan, Q., Guguin, J., Jouanno, E., Herpin, A., ... Guiguen, Y. (2019). Sex chromosome and sex locus characterization in the goldfish, Carassius auratus. BioRxiv, 2019.12.20.875377. doi: 10.1101/2019.12.20.875377

Wessels, S., Krause, I., Floren, C., Schütz, E., Beck, J., \& Knorr, C. (2017). DdRADseq reveals determinants for temperature-dependent sex reversal in Nile tilapia on LG23. BMC Genomics, 18(1), 531. doi: 10.1186/s12864-017-3930-0

Whitehead, J. A., Benfey, T. J., \& Martin-Robichaud, D. J. (2012). Ovarian development and sex ratio of gynogenetic Atlantic cod (Gadus morhua). Aquaculture, 324-325, 174-181. doi: 10.1016/j.aquaculture.2011.10.039

Wilson, C. A., High, S. K., McCluskey, B. M., Amores, A., Yan, Y., Titus, T. A., ... Postlethwait, J. H. (2014). Wild sex in zebrafish: Loss of the natural sex determinant in domesticated strains. Genetics, 198(3), 1291-1308. doi: 10.1534/genetics.114.169284

Wu, C. I. (1983). The fate of autosomal modifiers of the sex-ratio trait in drosophila and other sexlinked meiotic drive systems. Theoretical Population Biology, 24(2), 107-120. doi: 10.1016/0040-5809(83)90035-7

Yano, A., Nicol, B., Jouanno, E., Quillet, E., Fostier, A., Guyomard, R., \& Guiguen, Y. (2013). The sexually dimorphic on the $\mathrm{Y}$-chromosome gene ( $\mathrm{sdY}$ ) is a conserved male-specific $\mathrm{Y}$ chromosome sequence in many salmonids. Evolutionary Applications, 6(3), 486-496. doi: 


\section{DATA ACCESSIBILITY}

All RAD-Sequencing experiments have been submitted to Genbank under the Bioproject PRJNA548074. A computational workflow implementing all the analyses performed in this study, including generating figures, is available at https://github.com/RomainFeron/paper-sexdeterminationradsex. RADSex is released under GPLv3 license; source code, installation instructions, and documentation are available at https://github.com/SexGenomicsToolkit/radsex. sgtr is released under GPLv3 license and available at https://github.com/SexGenomicsToolkit/sgtr.

\section{AUTHOR CONTRIBUTIONS}

RF designed and implemented RADSex and sgtr, with feedback from QP, MW, and YG. YG, JHP, and MSch designed the PhyloSex project and advised on results interpretation. QP, MW, BI, JA, AH, KK, ASR, KD, SK, CK, JHP, MSch and YG participated in the analysis of the results. HP and LJ prepared libraries and performed the sequencing. RF, QP, JHP, MSch and YG drafted the manuscript. RF, QP, RMW, JHP, MSch, and YG revised the manuscript. YG, MSch, JHP, EJ, SK, MW, MA, CW, BM, AA, TD, FWG, MK, HWD, MO, RN, TS, MN, MSt, AW, ØK and IB collected, sexed and/or extracted and prepared gDNA samples. All authors approved the final manuscript. 


\begin{tabular}{|c|c|c|c|}
\hline Species & $\begin{array}{l}\text { SD system identified } \\
\text { with radsex }\end{array}$ & $\begin{array}{c}\text { Number of sex } \\
\text { markers (min depth) }\end{array}$ & $\begin{array}{c}\text { SD system previously } \\
\text { described }\end{array}$ \\
\hline Cyprinus carpio & $\mathrm{XX} / \mathrm{XY}$ with male outliers & $7(d=10)$ & XX/XY (Gomelsky, 2003) \\
\hline Danio aesculapii & No SD identified & $0(d=10)$ & Unknown \\
\hline Danio albolineatus & No SD identified & $0(d=10)$ & Unknown \\
\hline Danio choprae & No SD identified & $0(d=10)$ & Unknown \\
\hline Danio kyathit & No SD identified & $0(d=10)$ & Unknown \\
\hline Gadus morhua & $\mathrm{XX} / \mathrm{XY}$ with outliers & $3(d=10)$ & $\mathrm{XX} / \mathrm{XY}$ (Haugen et al., 2012) \\
\hline Gymnocorymbus ternetzi & No SD identified & $0(d=2)$ & $\begin{array}{c}\text { Potentially XX/XY (David \& } \\
\text { Pandian, 2016) }\end{array}$ \\
\hline Gymnotus carapo & $\mathrm{XX} / \mathrm{XY}$ with female outliers & $8(d=10)$ & $\overline{X X / X Y}$ (da Silva et al., 2014) \\
\hline Hippocampus abdominalis & No SD identified & $0(d=10)$ & Unknown \\
\hline Lepisosteus oculatus & No SD identified & $0(d=2)$ & Unknown \\
\hline Notothenia rossii & No SD identified & $0(d=10)$ & Unknown \\
\hline Plecoglossus altivelis & $\mathbf{X X / X Y}$ & $47(d=10)$ & $\overline{X X / X Y}$ (Watanabe et al., 2004) \\
\hline Poecilia sphenops & $\mathrm{XX} / \mathrm{XY}$ with female outliers & $7(d=10)$ & $\begin{array}{l}\text { ZZ/ZW (Nanda et al., 1993) } \\
\text { XX/XY (Volff \& Schartl, 2001) }\end{array}$ \\
\hline Sander vitreus & No SD identified & $0(d=2)$ & Unknown \\
\hline Tinca tinca & $\mathbf{X X / X Y}$ & $6(d=10)$ & Unknown \\
\hline
\end{tabular}

Table 1: Summary of RADSex results for the $\mathbf{1 5}$ datasets analyzed. Species where a sexdetermination system was identified with radsex are highlighted with a grey background. The number of markers significantly associated with phenotypic sex is also given for each species, 
with the value of ' $d$ ' indicating the minimal number of reads for a marker to be considered present in an individual in the analysis. 
Figure 1: Typical RADSex workflow for sex determination system identification.

The process command from radsex computes a table containing the sequencing depth of each marker in each individual. This table is then used as input for the distrib command to compute the distribution of markers between males and females, and this distribution is visualized with the radsex_distrib() function of sgtr. Markers significantly associated with sex are extracted with the signif command and their depth in each individual is visualized with the radsex_markers_depth() function of sgtr. When a genome assembly is available, markers can be aligned with the map command and results are visualized with the radsex_map_circos() function of sgtr.

Figure 2: Tile plots showing the theoretical result of distrib in four scenarios. Each plot shows the distribution of RADSex markers between phenotypic males (horizontal axis) and phenotypic females (vertical axis), with color intensity for a tile indicating the number of markers present in the corresponding number of males and females. Tiles for which association with phenotypic sex is significant (Chi-squared test, $p<0.05$ after Bonferroni correction) are highlighted with a red border. A: Tile plot for an $X X / X Y$ sex determination (SD) system showing markers present in all males and absent from all females in the bottom right corner. B: Tile plot for a ZZ/ZW SD system showing markers present in all females and absent from all males in the top left corner. C: Tile plot for an XX/XY SD system with five male outliers showing markers present in all but five males and absent from all females at position $(15,0)$. D: Tile plot for a ZZ/ZW SD system with two male and three female outliers showing markers present in all but three females and absent from all but two males at position $(2,17)$.

Figure 3: Performance of the RADSex workflow (process, signif, and distrib). A: Total runtime of RADSex (in minutes) plotted against the number of RAD-seq reads for each dataset. B: Peak memory of RADSex in gigabytes plotted against the number of RAD-seq reads for each dataset.

Figure 4: Validation of the RADSex approach on the Oryzias latipes dataset. A: Tile plot showing the distribution of RADSex markers between males (horizontal axis) and females (vertical axis) using the original sex assignment and a minimum depth of $10(\mathrm{~d}=10)$. Color intensity indicates the number of markers present in the corresponding number of males and females, and a red border indicates significant association with sex (Chi-squared test, $p<0.05$ after Bonferroni correction). B: Heatmap showing individual sequencing depth for all markers present in at least 20 males and absent $(\mathrm{d}=10)$ from all females. Each row corresponds to a marker, and each column corresponds to an individual. Males (blue labels) and females (red labels) clustered in two separate groups, except for two male outliers, identified by the green arrows, which clustered with the females. In addition, six males forming a sub-cluster within the male cluster had low or null depth for half of the markers. C: Manhattan plot showing $-\log 10(P)$ of a chi-squared test on number of males and number of females, after Bonferroni correction, for all RADSex markers aligned to the genome of $O$. latipes, with odd linkage groups (LGs) in yellow with light background and even LGs in blue with shaded background. Unplaced scaffolds were concatenated in a super scaffold $(U)$ by order of decreasing size. The dashed line indicates a p-value of 0.05 after Bonferroni correction. Most markers significantly associated with sex were aligned to LG01 (Ola1) or to unplaced scaffolds. D: The $-\log 10(\mathrm{P})$ of a chi-squared test on the number of males and number of females is plotted against genomic position on LG01 of $O$. latipes (Ola1) for all RADSex markers aligned to this LG. The dashed line indicates a p-value of 0.05 after Bonferroni correction. Markers significantly associated with sex were aligned to a continuous region between 14.7 and $31.9 \mathrm{Mb}$. Markers present in all males apart from the two outliers and absent in all females were aligned in a continuous region spanning from 17 to $23 \mathrm{Mb}$ (green box), while male-biased markers absent from the sub-cluster of six males were aligned in two regions, from 14 to $17 \mathrm{Mb}$ and from 24 to $31.9 \mathrm{Mb}$ (purple boxes). 
Figure 5: Tile plots showing the distribution of markers between males and females for six species (A-F) with markers significantly associated with sex. The number of markers, represented by tile color intensity, is plotted for every combination of number of males (horizontal axis) and number of females (vertical axis) for each species. Combinations of number of males and number of females for which association with sex is significant $(p<0.05$, Chi-squared test with Bonferroni correction) are highlighted in red. Distributions were computed with a minimum depth of $10(-d 10)$.

Figure 6: Results of RADSex analysis on Gadus morhua. A: Tile plot showing the presence of markers in each individual for all markers present with depth higher than five in at least 20 males and in fewer than two females. Each row corresponds to a marker and each column corresponds to an individual, with females labeled in red and males labeled in blue. B: Manhattan plot showing - $\log 10(\mathrm{P})$ of a chi-squared test on the number of males and number of females for all RADSex markers aligned to the genome of G. morhua, with odd linkage groups (LGs) in yellow with light background and even LGs in blue with shaded background. Unplaced scaffolds were concatenated in a super scaffold $(U)$ by decreasing order of size. The dashed line indicates a $p$ value of 0.05 after Bonferroni correction. C: $-\log 10(\mathrm{P})$ of a chi-squared test on the number of males and the number of females for all RADSex markers aligned to LG11 of G. morhua. The dashed line indicates a $p$-value of 0.05 after Bonferroni correction. Markers significantly associated with sex $(p<0.05)$ and other markers strongly associated with sex were aligned to the first $20 \mathrm{Mb}$ of the chromosome. The position of the candidate master sex determining gene is indicating by a red arrow. 


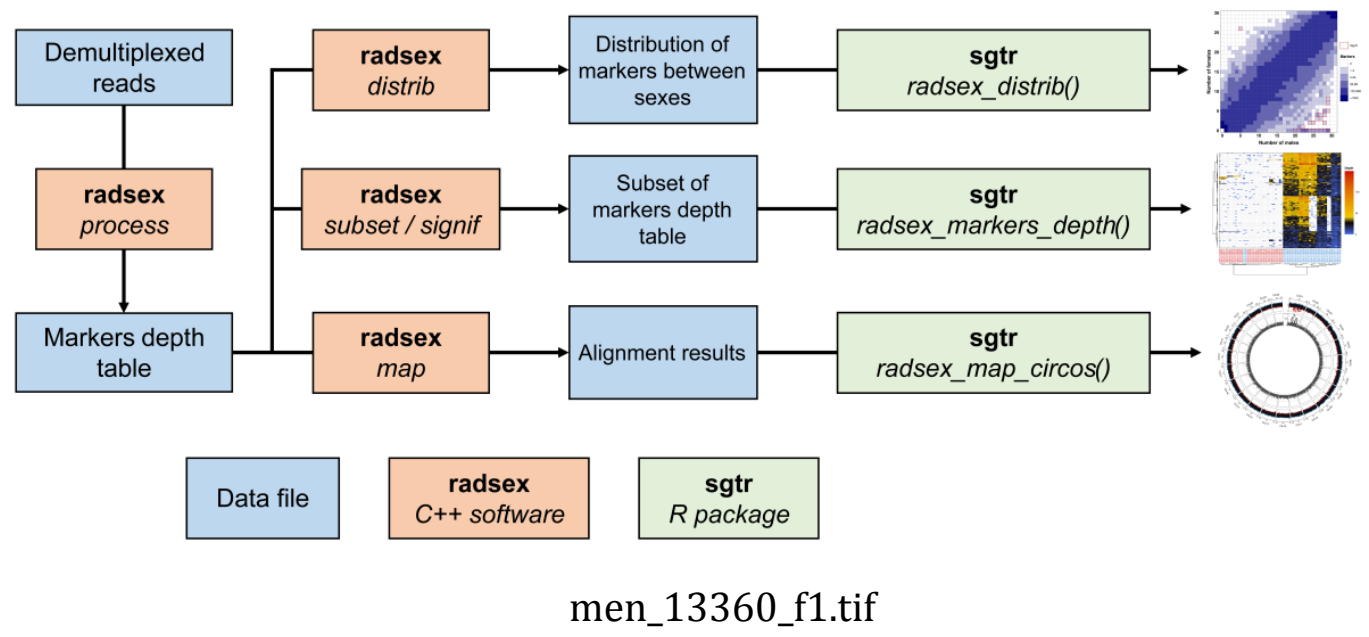

This article is protected by copyright. All rights reserved 

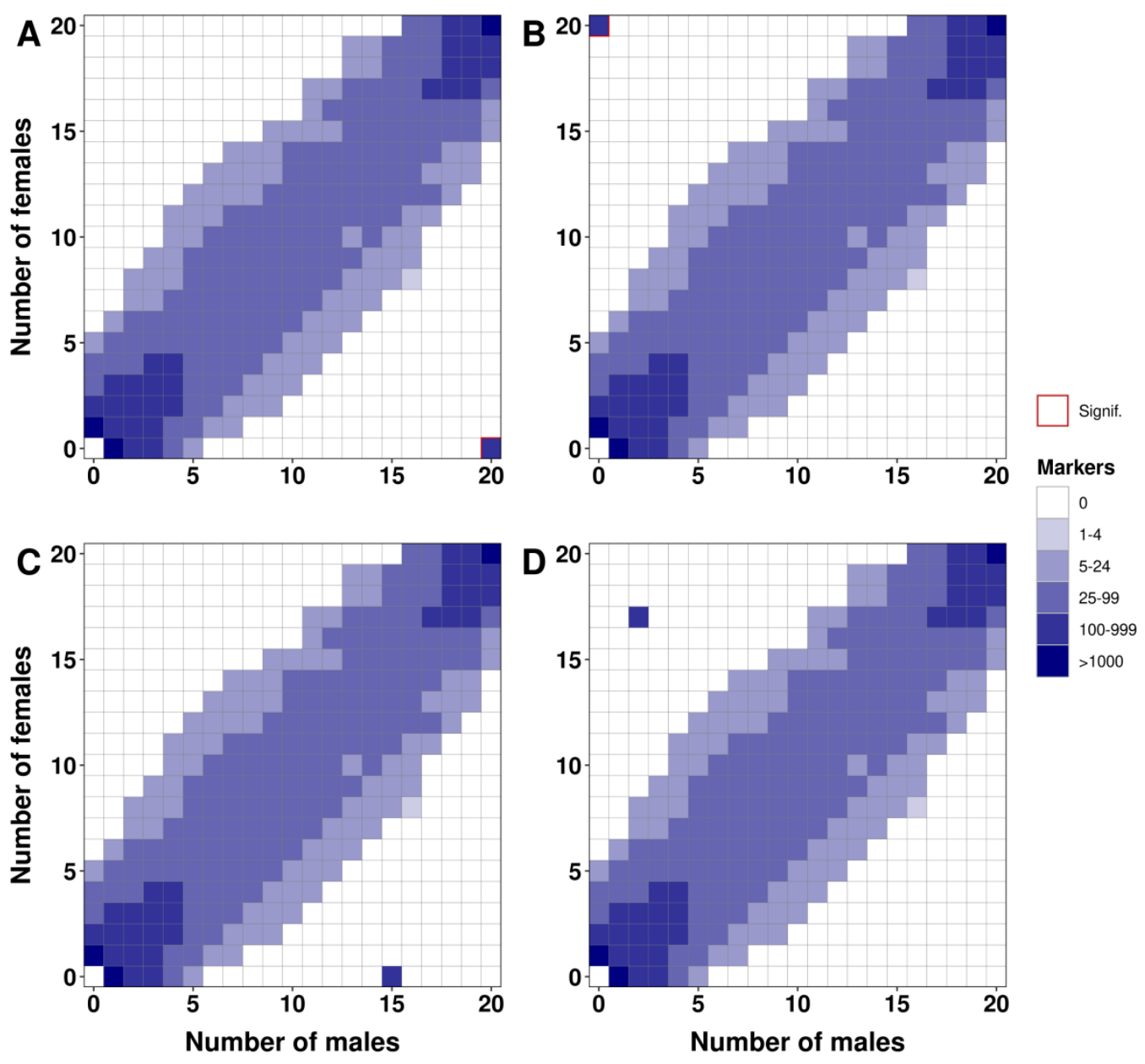

men_13360_f2.tif

This article is protected by copyright. All rights reserved 


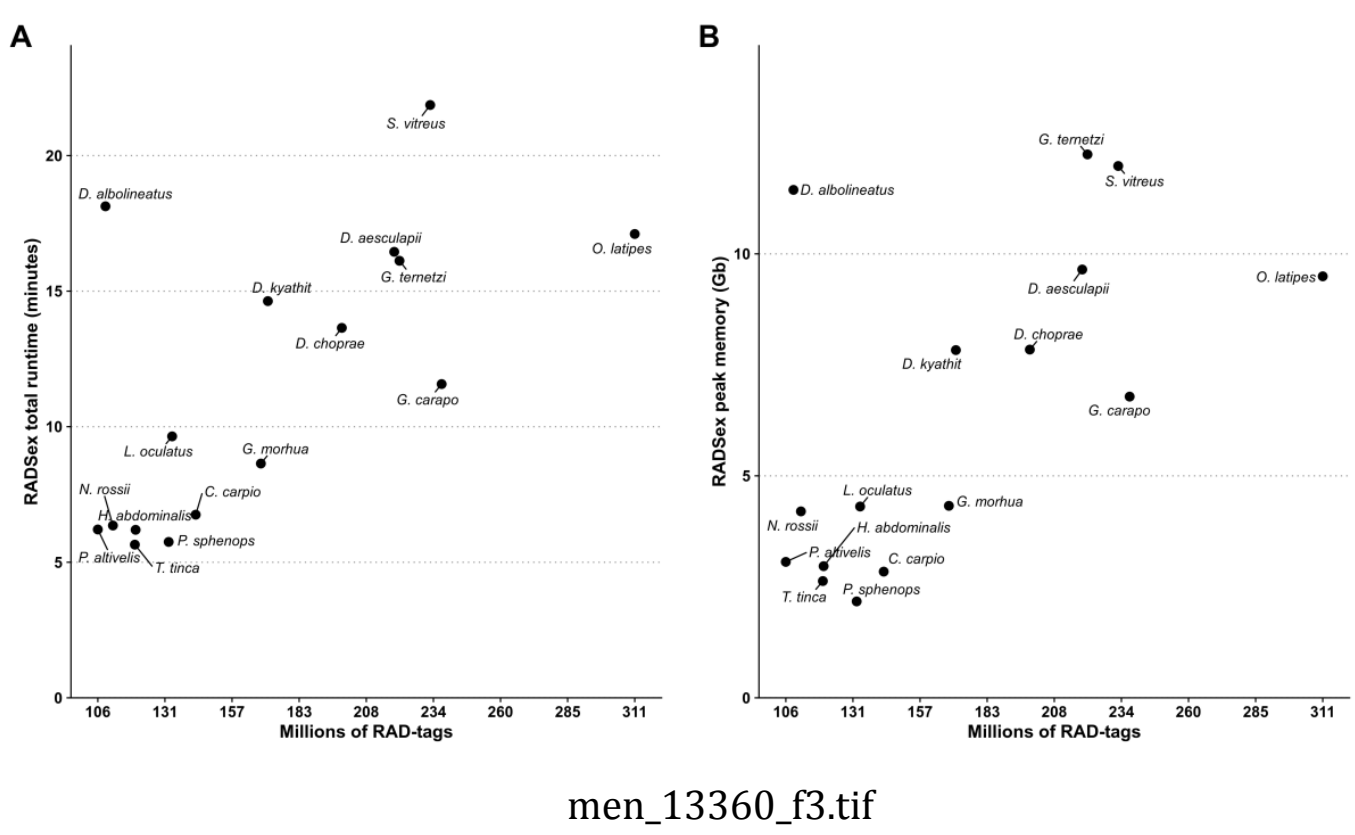

This article is protected by copyright. All rights reserved 

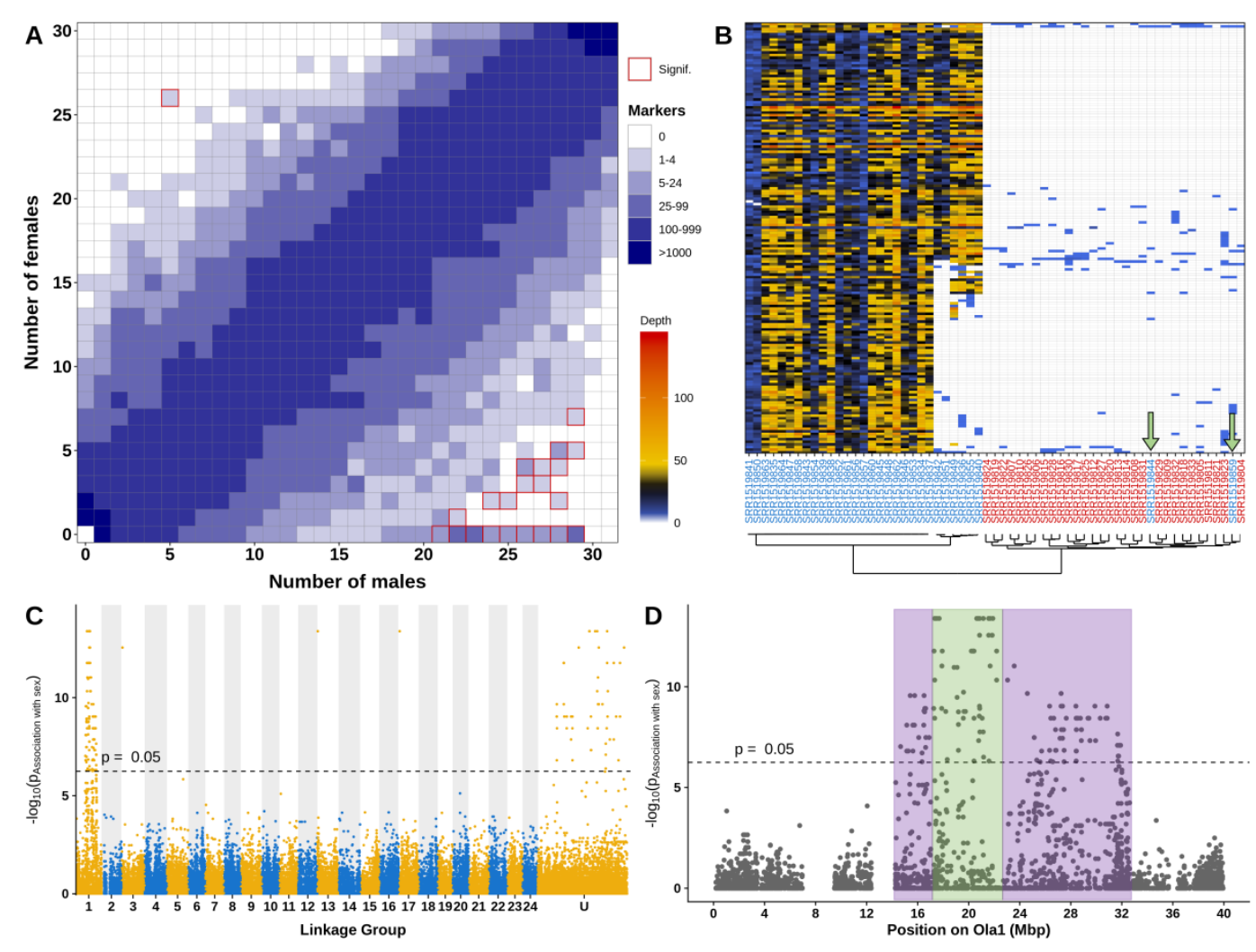

men_13360_f4.tif 

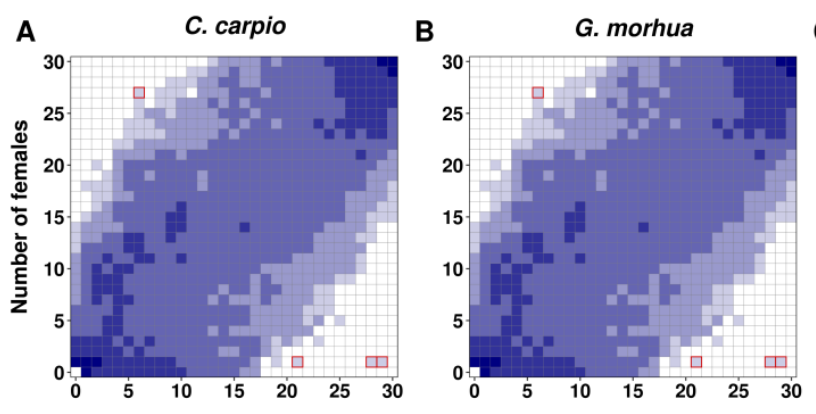

C G. carapo
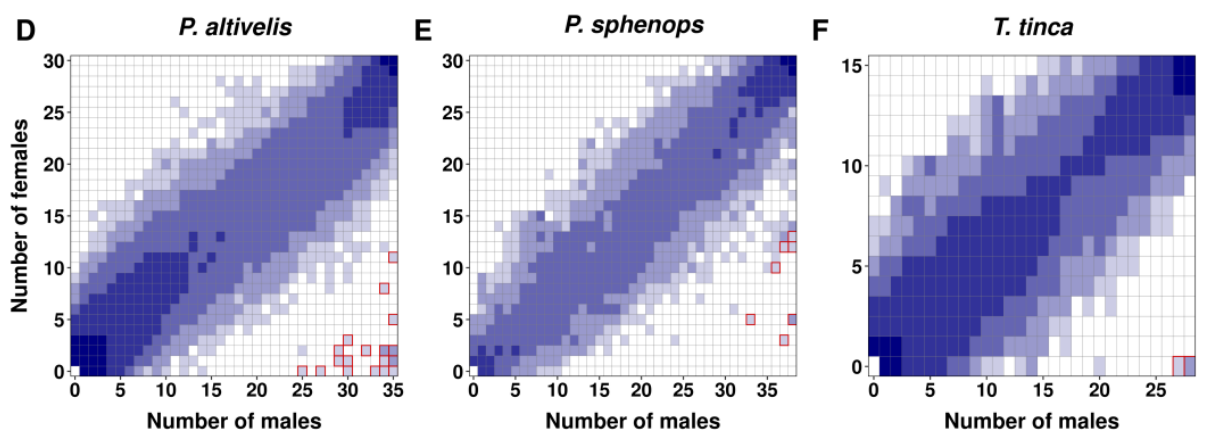

men_13360_f5.tif 

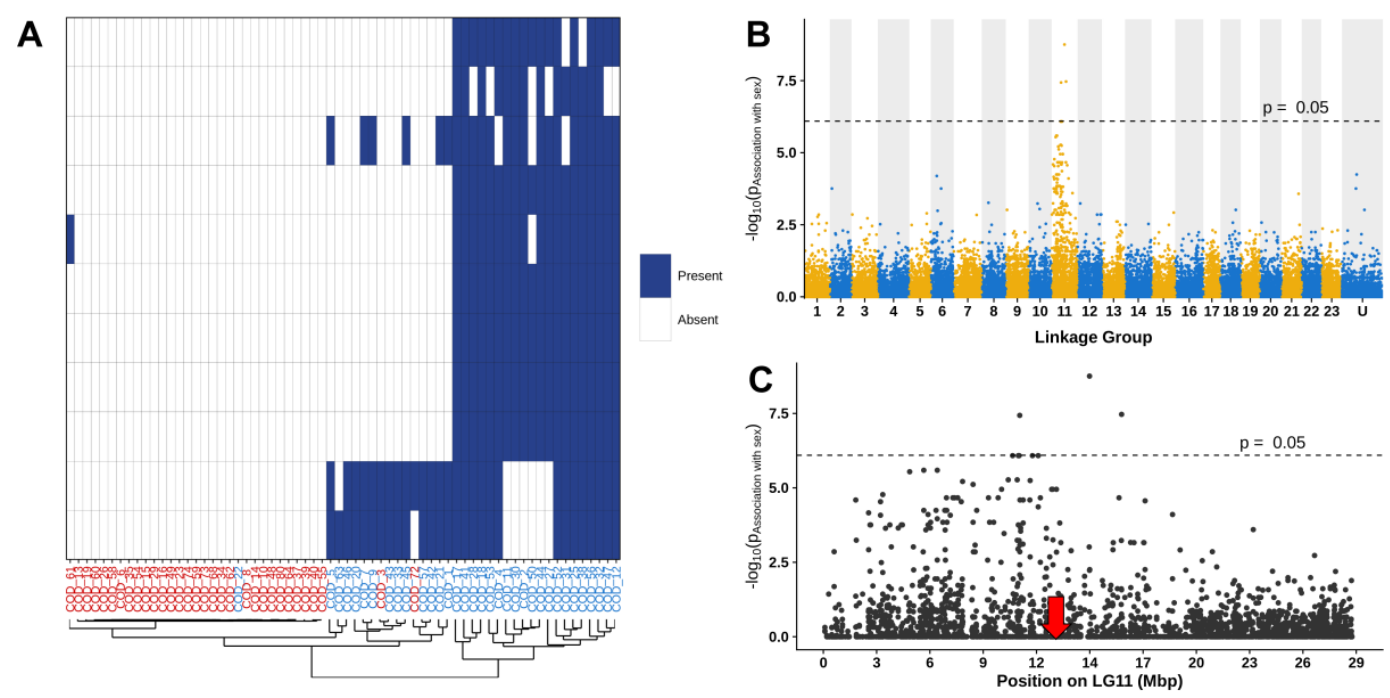

men_13360_f6.tif

This article is protected by copyright. All rights reserved 